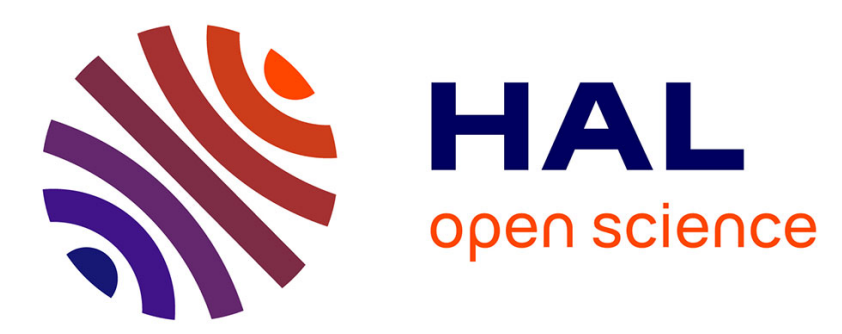

\title{
Consistent Discretization of Finite-time and Fixed-time Stable Systems
}

Andrey Polyakov, Denis Efimov, Bernard Brogliato

\section{To cite this version:}

Andrey Polyakov, Denis Efimov, Bernard Brogliato. Consistent Discretization of Finite-time and Fixed-time Stable Systems. SIAM Journal on Control and Optimization, 2019, 57 (1), pp.78-103. 10.1137/18M1197345. hal-01838712v2

\section{HAL Id: hal-01838712 https://hal.inria.fr/hal-01838712v2}

Submitted on 9 Nov 2018

HAL is a multi-disciplinary open access archive for the deposit and dissemination of scientific research documents, whether they are published or not. The documents may come from teaching and research institutions in France or abroad, or from public or private research centers.
L'archive ouverte pluridisciplinaire HAL, est destinée au dépôt et à la diffusion de documents scientifiques de niveau recherche, publiés ou non, émanant des établissements d'enseignement et de recherche français ou étrangers, des laboratoires publics ou privés. 


\title{
Consistent Discretization of Finite-time and Fixed-time Stable Systems *
}

\author{
Andrey Polyakov ${ }^{\dagger}$, Denis Efimov ${ }^{\dagger}$, and Bernard Brogliato ${ }^{\ddagger}$
}

Abstract. Algorithms of implicit discretization for generalized homogeneous systems having a possible discontinuity only at the origin are developed. They are based on the transformation of the original system to an equivalent one which admits an implicit or a semi-implicit discretization schemes preserving the stability properties of the continuous-time system. Namely, the discretized model remains finite-time stable (in the case of negative homogeneity degree), and practically fixed-time stable (in the case of positive homogeneity degree). The theoretical results are supported with numerical examples.

1. Introduction. Discretization issues are important for a digital implementation of estimation and control algorithms as well as for a computer simulation of control processes. Construction of a consistent stable discretization is complex for essentially non-linear ordinary differential equations (ODEs), which do not satisfy some classical regularity assumptions. An inconsistent discretization of non-Lipschitz feedback algorithms results in degradation of control precision [1], chattering effect [2], or even instability [3]. For example, the sliding mode algorithms are known to be difficult in practical realization [1], [4], [5] due to discontinuous (set-valued) nature, which may involve chattering caused by the discretization (the so-called numerical chattering). The mentioned papers have discovered that the implicit discretization technique is useful for practical implementation of non-smooth and discontinuous control and estimation algorithms. In particular, chattering suppression in both input and output, as well as a good closed-loop performance have been confirmed experimentally in [1], [6], [7].

Finite-time stability is a desirable property for many control and estimation algorithms [2], [8], [9], [10], [11], [12]. It means that system's trajectories reach a stable equilibrium (or a set) in a finite time, in contrast to asymptotic stability allowing this only for the time tending to infinity. If the settling (reaching) time is globally bounded for all initial conditions then the origin is fixed-time stable (see, e.g. [13]). The corresponding ODE models do not satisfy the Lipschitz condition, at least at the origin. In the general case, an application of the conventional implicit or explicit discretization schemes does not guarantee that finitetime or fixed-time stability properties will be preserved [14], [15], [16]. The latter means that the discrete-time model may be inconsistent with the continuous-time one. However, the discretized systems may remain globally finite-time stable in some cases (see [1], [4], [17], [18]). The aim of this paper is to study systematically the problem of consistent discretization of the so-called generalized homogeneous non-linear systems. The discretized model is consistent if it preserves the stability property (e.g., exponential, finite-time or fixed-time stability) of the original continuous-time system.

Homogeneity is a certain form of symmetry studied in systems and control theory [10], [19],

\footnotetext{
*The preliminary version of this work, dealing only with the finite-time stability case without proofs, has been presented at the 15th International Workshop on Variable Structure Systems and Sliding Mode Control, 2018.

†Inria, Univ. Lille, CNRS, UMR 9189 - CRIStAL, F-59000 Lille, France (andrey.polyakov@inria.fr, denis.efimov@inria.fr) and ITMO University, 197101, Saint-Petersburg, Russia.

${ }^{\ddagger}$ Univ. Grenoble Alpes, Inria, CNRS, Grenoble INP, LJK, Grenoble, 38000, France (bernard.brogliato@inria.fr).
} 
[20], [21],[22], [23], [24]. The standard homogeneity, introduced originally by L. Euler in 17th century, is the symmetry of a mathematical object $f$ (e.g., function, vector field, operator, etc) with respect to the uniform dilation of the argument $x \rightarrow \lambda x$, namely, $f(\lambda x)=\lambda^{1+\nu} f(x), \lambda>0$ where $\nu \in \mathbb{R}$ is the so-called homogeneity degree. The type of homogeneity is identified by the corresponding dilation. For example, in [25], [26], [27] the uniform dilation is utilized but the articles [19], [28], [29] deal with the so-called weighted dilation: $x \rightarrow\left(\lambda^{r_{1}} x_{1}, \lambda^{r_{2}} x_{2}, \ldots, \lambda^{r_{m}} x_{n}\right)$, $r_{i}>0$. Nonlinear homogeneous differential equations and inclusions form an important class of control systems [10], [30], [31], [32]. They appear as local approximations [10], [20] or setvalued extensions [28], [29] of nonlinear systems, and include models of process control [33], nonholonomic systems [34], mechanical models with friction [28], etc. Time-optimal [35] and high order sliding mode algorithms [2], [29], [36] are homogeneous and finite-time convergent. Consistent discretization of such systems is an important problem for both control theory and practice.

The generalized homogeneity to be considered in this paper was introduced originally in [23] for infinite dimensional models such as partial differential equations (PDEs). It considers a strongly continuous group of linear bounded operators generated by a possibly unbounded linear operator as a dilation in a Banach space. A lot of well-known PDEs are homogeneous in thr generalized sense, e.g. heat, wave, Navier-Stocks, Saint-Venant, Korteweg-de Vries, fast diffusion equations. This paper deals with the finite-dimensional models of generalized homogeneous systems represented by ODEs. In [37], [38] it has been proved that any generalized homogeneous ODE is topologically equivalent (homeomorphic on $\mathbb{R}^{n}$ and diffeomorphic on $\left.\mathbb{R}^{n} \backslash\{\mathbf{0}\}\right)$ to a standard homogeneous one, and any asymptotically stable homogeneous system is topologically equivalent to a quadratically stable system. These two key facts are essentially used in the present paper.

Notice that an asymptotically stable homogeneous system is finite-time stable in the case of negative homogeneity degree [25], [28], [29], [38], [39] and practically fixed-time stable in the case of positive homogeneity degree [10], [13], [40]. Practical fixed-time stability implies that for any neighborhood of the origin there exists a time $T_{\max }$, such that independently of the initial condition, any trajectory of the system belongs to this neighborhood after the time $T_{\max }$.

In this paper we develop consistent discretization schemes, which allow the mentioned stability properties to be preserved for discretized models of generalized homogeneous systems. We demonstrate an efficiency of this scheme for several finite-time and fixed-time stable control systems. We also present results of numerical simulations confirming a complete elimination of the numerical chattering by means of the proposed discretization technique.

The basic ideas of further constructions are explained in the next two motivating examples.

1.1. Finite-time Stable Implicit Discretization. Let us consider two scalar non-linear control systems:

$$
\left\{\begin{array} { l } 
{ \dot { x } = u ( x ) } \\
{ u ( x ) = - 2 \sqrt { | x | } \operatorname { s g n } ( x ) , \quad y = \sqrt { | x | } \operatorname { s g n } ( x ) }
\end{array} \quad \left\{\begin{array}{l}
\dot{y}=\tilde{u}(y), \\
\tilde{u}(y) \in-\operatorname{sgn}(y),
\end{array}\right.\right.
$$


where

$$
\operatorname{sgn}(\rho)=\left\{\begin{array}{ccc}
1 & \text { if } & \rho>0 \\
{[-1,1]} & \text { if } & \rho=0 \\
-1 & \text { if } & \rho<0 .
\end{array}\right.
$$

Both systems are finite-time stable, i.e., the state of each system vanishes in a finite time. These two systems are topologically equivalent (homeomorphic on $\mathbb{R}$ and diffeomorphic on $\mathbb{R} \backslash\{\mathbf{0}\})$. More precisely, if $x\left(\cdot, x_{0}\right)$ is the solution of the first system with $x(0)=x_{0} \in \mathbb{R}$ then $y\left(\cdot, y_{0}\right)=\sqrt{\left|x\left(\cdot, x_{0}\right)\right|} \operatorname{sgn}\left(x\left(\cdot, x_{0}\right)\right)$ is the solution of the second system with $y(0)=y_{0}=$ $\sqrt{\left|x_{0}\right|} \operatorname{sgn}\left(x_{0}\right)$, and vice versa.

Both control systems admit an implicit Euler discretization (a sampled-time realization) as follows:

$$
\left\{\begin{array} { l } 
{ x _ { i + 1 } = x _ { i } + h u _ { i } } \\
{ u _ { i } = - 2 \sqrt { | x _ { i + 1 } | } \operatorname { s g n } ( x _ { i + 1 } ) }
\end{array} \Leftrightarrow \quad \Leftrightarrow \quad \left\{\begin{array}{l}
y_{i+1}=y_{i}+h \tilde{u}_{i} \\
\tilde{u}_{i} \in \operatorname{sgn}\left(y_{i+1}\right)
\end{array}\right.\right.
$$

where $h>0$ is the sampling period, $i=0,1,2 \ldots$ and $x_{i}=x\left(i h, x_{0}\right)\left(\operatorname{resp} . y_{i}=y\left(i h, x_{0}\right)\right)$ provided that $u(t)=u_{i}$ (resp. $\left.\tilde{u}(t)=\tilde{u}_{i}\right)$ for $t \in[i h,(i+1) h)$. Such discretizations are called implicit because the control input is defined implicitly, but can nevertheless be calculated at time $t=k h$ as shown next.

The topological equivalence between these two systems is destroyed after discretization. Indeed, for the first system one has

$$
\left\{\begin{array}{l}
x_{i+1}=\left(\sqrt{h^{2}+\left|x_{i}\right|}-h\right)^{2} \operatorname{sgn}\left(x_{i}\right) \neq 0, \quad \forall i \geq 0, \\
u_{i}=2\left(h-\sqrt{h^{2}+\left|x_{i}\right|}\right) \operatorname{sgn}\left(x_{i}\right),
\end{array}\right.
$$

provided that $x_{0} \neq 0$, but the implicit scheme for the second equation gives (see [4] for the details)

$$
\begin{aligned}
& y_{i+1}=\left\{\begin{array}{ccc}
y_{i}-h \operatorname{sgn}\left(y_{i}\right) & \text { if } & \left|y_{i}\right|>h, \\
0 & \text { if } & \left|y_{i}\right| \leq h,
\end{array}\right. \\
& u_{i}=\left\{\begin{array}{ccc}
-\operatorname{sgn}\left(y_{i}\right) & \text { if } & \left|y_{i}\right|>h, \\
-\frac{y_{i}}{h} & \text { if } & \left|y_{i}\right| \leq h .
\end{array}\right.
\end{aligned}
$$

Obviously, if $\left|x_{i}\right| \neq 0$, then

$$
\left|x_{i+1}\right| \neq 0 \quad \text { and } \quad\left|x_{i+1}\right|=\gamma\left(x_{i}\right)\left|x_{i}\right|
$$

with

$$
\gamma\left(x_{i}\right):=\left(\sqrt{\frac{h^{2}}{\left|x_{i}\right|}+1}-\sqrt{\frac{h^{2}}{\left|x_{i}\right|}}\right)^{2} \in(0,1), \quad i=1,2, \ldots .
$$

In other words, the implicit discretization of the first equation is just asymptotically stable, but the implicit discretization of the second equation remains finite-time stable, i.e., there exists $i^{*}$ depending on $y_{0}$ and $h$ such that $y_{i}=0$ for all $i \geq i^{*}$. We infer that the implicit discretization of the considered equivalent systems does not yield equivalent discrete-time models. 


$$
\begin{array}{ccc}
\dot{x}=f(x) & y=\Phi(x) & \dot{y}=\tilde{f}(y) \\
\uparrow \begin{array}{c}
\text { a consistent } \\
\text { discrete-time } \\
\text { approximation }
\end{array} & \stackrel{\substack{\text { a consistent } \\
\text { discrete-time } \\
\text { approximation }}}{\Leftrightarrow} \downarrow \\
x_{i+1}=\Phi^{-1}\left(\Phi\left(x_{i}\right)+h \tilde{f}\left(\Phi\left(x_{i+1}\right)\right)\right) & \stackrel{x=\Phi^{-1}(y)}{\Leftrightarrow} & y_{i+1}=y_{i}+h \tilde{f}\left(y_{i+1}\right)
\end{array}
$$

Figure 1.1: The proposed scheme of the consistent implicit discretization of $\dot{x}=f(x)$

Therefore, a reasonable way to discretize consistently the first (continuous) system is to use its equivalence with the second (discontinuous) one. Indeed, using solutions of the discretized second system we can recover the finite-time convergent solutions of the first dynamics by means of posterior transformation of coordinates $x=y^{2} \operatorname{sgn}(y)$. The proposed scheme is depicted in Fig. 1.1, where $\Phi$ denotes a coordinate transformation to an equivalent system. It gives the following discrete-time model for the first system

$$
\begin{aligned}
& \hat{x}_{i+1}=\left\{\begin{array}{ccc}
\left(\sqrt{\left|\hat{x}_{i}\right|}-h\right)^{2} \operatorname{sgn}\left(\hat{x}_{i}\right) & \text { if } & \left|\hat{x}_{i}\right|>h^{2}, \\
0 & \text { if } & \left|\hat{x}_{i}\right| \leq h^{2},
\end{array}\right. \\
& \hat{u}_{i}=\left\{\begin{array}{cll}
\left(h-2 \sqrt{\left|\hat{x}_{i}\right|}\right) \operatorname{sgn}\left(\hat{x}_{i}\right) & \text { if } & \left|\hat{x}_{i}\right|>h^{2}, \\
-\frac{\hat{x}_{i}}{h} & \text { if } & \left|\hat{x}_{i}\right| \leq h^{2} .
\end{array}\right.
\end{aligned}
$$

with $\hat{x}_{0}=x_{0}$. This system is expected to be finite-time stable. Indeed, if $\left|\hat{x}_{0}\right|>h^{2}$ then

$$
\left|\hat{x}_{i+1}\right|=\hat{\gamma}\left(\hat{x}_{i}\right)\left|\hat{x}_{i}\right| \quad \text { with } \quad \hat{\gamma}\left(\hat{x}_{i}\right):=\left(1-\frac{h}{\sqrt{\left|\hat{x}_{i}\right|}}\right)^{2}<\hat{\gamma}\left(\hat{x}_{0}\right) \in(0,1), \quad i=1,2, \ldots,
$$

and there exists $i_{h} \in \mathbb{N}$ such that $\left|\hat{x}_{i_{h}}\right| \leq h^{2}$, i.e., $x_{i_{h}+1}=0$.

This example motivates us to conjecture that even continuous finite-time stable systems may have a consistent discrete-time approximation that preserves the finite-time stability property. A design of the corresponding discretization scheme is expected to be based on the transformation of the original system to an equivalent one, which admits a finite-time stable implicit discretization.

1.2. Practically Fixed-time Stable Semi-Implicit Discretization. Let us consider now the scalar system

$$
\dot{x}=-|x| x \text {. }
$$

It is easy to show that for any $\varepsilon>0$, each solution of the latter system satisfies $\left|x\left(t, x_{0}\right)\right|<\varepsilon$ for $t>\frac{1}{\varepsilon}$ independently of the initial state $x_{0}$, i.e., the origin of (1.1) is practically fixed-time stable. 
The explicit Euler discretization of the system (1.1)

$$
x_{i+1}=x_{i}-h\left|x_{i}\right| x_{i}
$$

is not appropriate in practice. It is locally conditionally stable and its solutions blow up if $h>2 /\left|x_{0}\right|$, i.e., for sufficiently large initial conditions. Moreover, the discretized model has oscillatory discrete-time solutions if $\left|x_{0}\right| \in\left(h^{-1}, 2 h^{-1}\right)$ and finite-time time convergence to zero if $\left|x_{0}\right|=h^{-1}$, while the trajectories of the continuous-time system are monotone and reach zero only as time tends to infinity (for $x_{0} \neq 0$ ).

The implicit Euler discretization given by $x_{i+1}=x_{i}-h\left|x_{i+1}\right| x_{i}$ yields the equation

$$
x_{i+1}=\frac{\sqrt{1+4 h\left|x_{i}\right|}-1}{2 h} \operatorname{sgn}\left(x_{i}\right),
$$

which has monotone solutions converging asymptotically to zero. However, this scheme does not preserve practical fixed-time stability. Thus the discrete-time model given by the fully implicit Euler scheme is inconsistent with the continuous-time model in the context of the decay rate.

Using a semi-implicit Euler discretization for the considered system, we derive

$$
\frac{x_{i+1}-x_{i}}{h}=-\left|x_{i}\right| x_{i+1}
$$

or, equivalently,

$$
x_{i+1}=\frac{x_{i}}{1+h\left|x_{i}\right|} .
$$

It is easy see that $\left|x_{1}\right| \leq(h)^{-1}$ independently of $x_{0}$, but

$$
\begin{gathered}
\left|x_{2}\right|=\frac{1}{\left|x_{1}\right|^{-1}+h} \leq \frac{1}{h+h}=(2 h)^{-1} \\
\left|x_{3}\right|=\frac{1}{\left|x_{2}\right|^{-1}+h} \leq \frac{1}{2 h+h}=(3 h)^{-1} \\
\left|x_{i}\right| \leq(i h)^{-1}
\end{gathered}
$$$$
\cdots
$$

i.e., the origin of the obtained discrete-time system is practically fixed-time stable. Below we extend this semi-implicit discretization scheme to generalized homogeneous systems with positive homogeneity degrees.

1.3. Notation. $\mathbb{R}$ is the field of real numbers; $\mathbb{R}_{+}=\{x \in \mathbb{R}: x>0\} ; \mathbb{N}$ is the set of natural numbers; $\|\cdot\|$ denotes a norm in $\mathbb{R}^{n}$ and

$$
\|A\|=\sup _{x \in \mathbb{R}^{n}} \frac{\|A x\|}{\|x\|} \text { and }\lfloor A\rfloor=\inf _{x \in \mathbb{R}^{n}} \frac{\|A x\|}{\|x\|} \text { if } A \in \mathbb{R}^{n \times n} ;
$$

$S$ denotes a unit sphere in $\mathbb{R}^{n}$ and $\mathcal{B}$ denotes a unit ball in $\mathbb{R}^{n} ; C^{n}(X, Y)$ is the set of continuously differentiable (at least up to the order $n$ ) maps $X \rightarrow Y$, where $X, Y$ are open subsets of finite dimensional Euclidean spaces; $\frac{\partial}{\partial x}=\left(\frac{\partial}{\partial x_{1}}, \ldots, \frac{\partial}{\partial x_{n}}\right) ; I_{n} \in \mathbb{R}^{n \times n}$ is the identity matrix; $\mathbf{0}$ denotes the zero element, e.g. $\mathbf{0} \in \mathbb{R}^{n}$ is the zero vector but $\mathbf{0} \in \mathbb{R}^{n \times n}$ is the zero 
matrix; $\operatorname{diag}\left\{\lambda_{1}, \ldots, \lambda_{n}\right\}$ is the diagonal matrix with elements $\lambda_{i}$; the order relation $P \succ 0$ means positive definiteness of the symmetric matrix $P \in \mathbb{R}^{n \times n} ; \lambda_{\max }(P)$ and $\lambda_{\min }(P)$ denote maximal and minimal eigenvalues of the symmetric matrix $P \in \mathbb{R}^{n \times n} ; \Re(\lambda)$ denotes the real part of the complex number $\lambda$; the notation $P^{\frac{1}{2}}$ means that $P^{\frac{1}{2}}=M$ is such that $P=M^{2}$; a function $c:[0,+\infty) \rightarrow[0,+\infty)$ belongs to the class $\mathcal{K}$ if it is continuous, monotone increasing and $c(0)=0$. A set-valued mapping is a mapping $\mathbb{R}^{n} \rightrightarrows \mathbb{R}^{m}$, which associates with each $x \in \mathbb{R}^{n}$, a set of $\mathbb{R}^{m}$.

1.4. Structure of the article. This article is organized as follows. First, the problem statement and basic assumptions are presented. Next, some preliminary results and related definitions for generalized homogeneous systems are considered. The consistent discretization schemes for finite-time and practically fixed-time stable homogeneous systems are proposed in Sections 4 and 5. Numerical examples are given in Section 6. Finally, some potential applications of the obtained results in control theory, as well as some promising directions of future research, are discussed.

2. Problem Statement. Let us consider the non-linear system

$$
\dot{x}=f(x), \quad t>0, \quad x(0)=x_{0},
$$

where $x(t) \in \mathbb{R}^{n}$ is the system state and the nonlinear function $f: \mathbb{R}^{n} \rightarrow \mathbb{R}^{n}$ is continuous on $\mathbb{R}^{n} \backslash\{0\}$, i.e., the only possible discontinuity point of $f$ is the origin. The system (2.1) is assumed to be forward complete for solutions understood in the sense of Filippov [41]:

An absolutely continuous function $\phi\left(\cdot, x_{0}\right):[0,+\infty) \rightarrow \mathbb{R}^{n}$ is a solution to (2.1) if $\phi\left(0, x_{0}\right)=x_{0}$ and for almost all $t>0$ it satisfies the differential inclusion

$$
\dot{x} \in F(x)=\bigcap_{\varepsilon>0} \bigcap_{\mu(N)=0} \operatorname{cof}(x+\varepsilon \mathcal{B} \backslash\{N\}),
$$

where $\mathcal{B}$ denotes the unit ball in $\mathbb{R}^{n}$, co denotes convex closure and $\mu(N)$ means that the Lebesgue measure of the set $N \subset \mathbb{R}^{n}$ is zero. In our case, $F(x)=\{f(x)\}$ is a singleton for $x \in \mathbb{R}^{n} \backslash\{\mathbf{0}\}$, but

$$
F(\mathbf{0})=\bigcap_{\varepsilon>0} \operatorname{cof}(\varepsilon B \backslash\{\mathbf{0}\})
$$

is a set, each time $f$ is discontinuous at $\mathbf{0}$.

2.1. Finite-Time Stable Systems. Recall [28], [42], [43] that the origin of the system (2.1) is said to be globally uniformly finite-time stable, if it is Lyapunov stable and there exists a locally bounded function $T: \mathbb{R}^{n} \rightarrow[0,+\infty)$ such that any solution $\phi\left(\cdot, x_{0}\right)$ to $(2.1)$ satisfies $\phi\left(t, x_{0}\right)=0$ for $t \geq T\left(x_{0}\right)$.

We understand the consistency of the discretization scheme for the finite-time stable system (2.1) in the sense of the following definition.

Definition 2.1. A (possibly) set-valued mapping

$$
Q: \mathbb{R}_{+} \times \mathbb{R}^{n} \times \mathbb{R}^{n} \rightrightarrows \mathbb{R}^{n}
$$

is said to be a consistent discrete-time approximation of the globally uniformly finite-time stable system (2.1) if 
- Existence property: for any $\tilde{x} \in \mathbb{R}^{n}$ and any $h>0$, there exists $\tilde{x}_{h} \in \mathbb{R}^{n}$ :

$$
\mathbf{0} \in Q\left(h, \tilde{x}, \tilde{x}_{h}\right),
$$

and $\tilde{x}_{h}=\mathbf{0}$ is the unique solution to $\mathbf{0} \in Q\left(h, \mathbf{0}, \tilde{x}_{h}\right)$.

- Finite-time convergence property: for any $h>0$ each sequence

$$
\left\{x_{i}\right\}_{i=0}^{+\infty}
$$

generated by the inclusion

$$
\mathbf{0} \in Q\left(h, x_{i}, x_{i+1}\right), \quad i=0,1,2, \ldots
$$

converges to zero in a finite number of steps, i.e., for any $x_{0} \in \mathbb{R}^{n} \backslash\{\mathbf{0}\}$ there exists $i^{*}>0$ such that

$$
x_{i}=\mathbf{0} \quad \text { for } \quad i \geq i^{*} .
$$

- Approximation property: for any $\varepsilon>0$ and any $R>\varepsilon$, there exists $\omega \in \mathcal{K}$ such that any sequence (2.4) generated by (2.5) satisfies

$$
\left\|\phi\left(h, x_{i}\right)-x_{i+1}\right\| \leq h \omega(h),
$$

provided that $\left\|x_{i+1}\right\|,\left\|x_{i}\right\| \in[\varepsilon, R]$, where $\phi\left(\cdot, x_{i}\right)$ is a solution to (2.1) with the initial condition $x(0)=x_{i}$.

Notice that the last property in this definition requires the existence of the conventional estimate (2.6) for the discretization error on any compact set from $\mathbb{R}^{n} \backslash\{\mathbf{0}\}$ (since $\varepsilon>0$ and $R>\varepsilon$ can be selected arbitrary small and arbitrary large, respectively). The origin is excluded because of a possible singularity of the vector field $f$ at zero (in particular, it can be discontinuous at the origin). The inequality (2.6) describes local (one-step) approximation error. An approximation error on the time interval $\left[0, T\left(x_{0}\right)\right]$ is $O(\omega(h))$ provided that $h=$ $T\left(x_{0}\right) / N$ with $N \in \mathbb{N}$. This error tends to zero as $h \rightarrow 0$, i.e., as $N \rightarrow+\infty$.

2.2. Practically Fixed-Time Stable Systems. The origin of the system (2.1) is said to be globally practically fixed-time stable if for any $r>0$ there exists $\bar{T}(r)>0$ such that any solution $\phi\left(\cdot, x_{0}\right)$ to $(2.1)$ satisfies $\left\|\phi\left(t, x_{0}\right)\right\| \leq r$ for $t \geq \bar{T}(r)$ independently of the initial condition [13]. The notion of consistent discretization of the practically fixed-time stable system can be introduced similarly.

Definition 2.2. A mapping $q: \mathbb{R}_{+} \times \mathbb{R}^{n} \times \mathbb{R}^{n} \rightarrow \mathbb{R}^{n}$ is said to be a consistent discrete-time approximation of the globally practically fixed-time stable system (2.1) if the existence and approximation properties (see Definition 2.1) hold and

- Practical fixed-time convergence property: for any $r>0$ there exists $N(r)>0$ such that any sequence

$$
\left\{x_{i}\right\}_{i=0}^{+\infty}, \quad x_{0} \neq \mathbf{0}
$$

generated by the equation

$$
q\left(h, x_{i}, x_{i+1}\right)=0, \quad i=0,1,2, \ldots
$$


satisfies

$$
\left\|x_{i}\right\| \leq r \quad \text { for } \quad i \geq N(r)
$$

independently of $x_{0}$.

The aim of the paper is to design a consistent (in the sense of Definitions 2.1 or 2.2) implicit discretization scheme for finite-time stable or practically fixed-time stable system (2.1), under the assumption that the vector field $f$ is homogeneous in the generalized sense [37].

\section{Preliminaries.}

3.1. Generalized Homogeneity. The generalized homogeneity [23], [37], [38], [44] deals with the group of linear transformations (linear dilations).

Definition 3.1. A map $\boldsymbol{d}: \mathbb{R} \rightarrow \mathbb{R}^{n \times n}$ is called dilation in $\mathbb{R}^{n}$ if it satisfies

- Group property: $\boldsymbol{d}(0)=I_{n}$ and $\boldsymbol{d}(t+s)=\boldsymbol{d}(t) \boldsymbol{d}(s)=\boldsymbol{d}(s) \boldsymbol{d}(t)$ for all $t, s \in \mathbb{R}$;

- Continuity property: $\boldsymbol{d}$ is a continuous map, i.e.,

$$
\forall t \in \mathbb{R}, \quad \forall \varepsilon>0, \quad \exists \delta=\delta(t, \varepsilon)>0 \quad: \quad|s-t|<\delta \quad \Rightarrow \quad\|\boldsymbol{d}(s)-\boldsymbol{d}(t)\| \leq \varepsilon ;
$$

- Limit property: $\lim _{s \rightarrow-\infty}\|d(s) x\|=0$ and $\lim _{s \rightarrow+\infty}\|d(s) x\|=+\infty$ uniformly on the unit sphere

$$
S:=\left\{x \in \mathbb{R}^{n}:\|x\|=1\right\} .
$$

The dilation $\mathbf{d}$ is a continuous group of invertible linear maps $\mathbf{d}(s) \in \mathbb{R}^{n \times n}, \mathbf{d}(-s)=$ $[\mathbf{d}(s)]^{-1}$. The matrix $G_{\mathbf{d}} \in \mathbb{R}^{n \times n}$

$$
G_{\mathbf{d}}=\lim _{s \rightarrow 0} \frac{\mathbf{d}(s)-I}{s}
$$

is known [45, Chapter 1] as the generator of the group $\mathbf{d}$. It satisfies the following properties

$$
\frac{d \mathbf{d}(s)}{d s}=G_{\mathbf{d}} \mathbf{d}(s) \quad \text { and } \quad \mathbf{d}(s)=e^{G_{\mathbf{d}} s}=\sum_{i=0}^{+\infty} \frac{s^{i} G_{\mathbf{d}}^{i}}{i !}, s \in \mathbb{R} .
$$

The latter implies $\mathbf{d}\left(s_{1}\right)-\mathbf{d}\left(s_{2}\right)=G_{\mathbf{d}} \int_{s_{2}}^{s_{1}} \mathbf{d}(s) d s, s_{1}, s_{2} \in \mathbb{R}$. The most popular dilations in $\mathbb{R}^{n}$ are [29], [28]

- uniform (or standard) dilation (L. Euler 17th century) :

$$
\mathbf{d}_{1}(s)=e^{s} I_{n}, \quad s \in \mathbb{R},
$$

- weighted dilation (Zubov 1958, [19]):

$$
\mathbf{d}_{2}(s)=\left(\begin{array}{cccc}
e^{r_{1} s} & 0 & \ldots & 0 \\
0 & e^{r_{2} s} & \cdots & 0 \\
\dddot{0} & \dddot{0} & \cdots & \dddot{r}_{\tilde{n}} s
\end{array}\right), s \in \mathbb{R}, r_{i}>0, i=1, \ldots, n
$$

They satisfy Definition 3.1 with $G_{\mathbf{d}_{1}}=I_{n}$ and $G_{\mathbf{d}_{2}}=\operatorname{diag}\left\{r_{i}\right\}$, respectively. In fact, any antiHurwitz ${ }^{1}$ matrix $G_{\mathbf{d}} \in \mathbb{R}^{n \times n}$ defines a dilation $\mathbf{d}(s)=e^{G_{\mathbf{d}} s}$ in $\mathbb{R}^{n}$. The geometric dilation studied in [21], [30] is more general since it allows the dilation group to be nonlinear.

\footnotetext{
${ }^{1}$ The matrix $G_{\mathbf{d}} \in \mathbb{R}^{n}$ is anti-Hurwitz if $-G_{\mathbf{d}}$ is Hurwitz.
} 
Definition 3.2. The dilation $\boldsymbol{d}$ is said to be strictly monotone if there exists $\beta>0$ such that $\|\boldsymbol{d}(s)\| \leq e^{\beta s}$ as $s<0$.

Obviously, the monotonicity of a dilation may depend on the norm $\|\cdot\|$ in $\mathbb{R}^{n}$.

Theorem 3.3. [38] Let $\boldsymbol{d}$ be a dilation in $\mathbb{R}^{n}$, then

1) all eigenvalues $\lambda_{i}$ of the matrix $G_{d}$ are placed in the right complex half-plane, i.e., $\Re\left(\lambda_{i}\right)>0, i=1,2, \ldots, n$;

2) there exists a matrix $P \in \mathbb{R}^{n \times n}$ such that

$$
P G_{\boldsymbol{d}}+G_{\boldsymbol{d}}^{\top} P \succ 0, \quad P=P^{\top} \succ 0
$$

3) the dilation $\boldsymbol{d}$ is strictly monotone with respect to the weighted Euclidean norm $\|\cdot\|=$ $\sqrt{\langle\cdot, \cdot\rangle}$ induced by the inner product $\langle x, z\rangle=x^{\top} P z$ with $P$ satisfying (3.2).

Moreover,

$$
\begin{aligned}
& e^{\alpha s} \leq\lfloor\boldsymbol{d}(s)\rfloor \leq\|\boldsymbol{d}(s)\| \leq e^{\beta s} \quad \text { if } \quad s \leq 0, \\
& e^{\beta s} \leq\lfloor\boldsymbol{d}(s)\rfloor \leq\|\boldsymbol{d}(s)\| \leq e^{\alpha s} \quad \text { if } \quad s \geq 0,
\end{aligned}
$$

where $\alpha=\frac{1}{2} \lambda_{\max }\left(P^{\frac{1}{2}} G_{\boldsymbol{d}} P^{-\frac{1}{2}}+P^{-\frac{1}{2}} G_{\boldsymbol{d}}^{\top} P^{\frac{1}{2}}\right)$ and $\beta=\frac{1}{2} \lambda_{\min }\left(P^{\frac{1}{2}} G_{\boldsymbol{d}} P^{-\frac{1}{2}}+P^{-\frac{1}{2}} G_{\boldsymbol{d}}^{\top} P^{\frac{1}{2}}\right)$.

The latter theorem proves that any dilation $\mathbf{d}$ is strictly monotone if $\mathbb{R}^{n}$ is equipped with the norm $\|x\|=\sqrt{x^{\top} P x}$, provided that the matrix $P \succ 0$ satisfies (3.2).

Definition 3.4. A continuous function $p: \mathbb{R}^{n} \rightarrow \mathbb{R}_{+}$is said to be $\boldsymbol{d}$-homogeneous norm if $p(x) \rightarrow 0$ as $x \rightarrow \mathbf{0}$ and $p(\boldsymbol{d}(s) x)=e^{s} p(x)>0$ for $x \in \mathbb{R}^{n} \backslash\{\mathbf{0}\}$ and $s \in \mathbb{R}$.

Obviously, the $\mathbf{d}$-homogeneous norm is neither a norm nor semi-norm in the general case, since the triangle inequality may not hold. However, many authors (see [10], [22], [44] and references therein) call a function satisfying the latter definition a homogeneous norm. We follow this tradition.

The canonical homogeneous norm $\|\cdot\|_{d}: \mathbb{R}^{n} \rightarrow \mathbb{R}_{+}$is defined as

$$
\|x\|_{\boldsymbol{d}}=e^{s_{x}} \quad \text { where } s_{x} \in \mathbb{R} \text { is such that }\left\|\boldsymbol{d}\left(-s_{x}\right) x\right\|=1 .
$$

The map $\|\cdot\|_{\mathbf{d}}: \mathbb{R}^{n} \rightarrow[0,+\infty)$ is well defined and single-valued for monotone dilations [44]. In [46] such a homogeneous norm was called canonical because it is induced by the (canonical) norm in $\mathbb{R}^{n}$. Notice that

$$
\left\lfloor\mathbf{d}\left(\ln \|x\|_{\mathbf{d}}\right)\right\rfloor \leq\|x\| \leq\left\|\mathbf{d}\left(\ln \|x\|_{\mathbf{d}}\right)\right\| \quad \text { for } \quad x \in \mathbb{R}^{n},
$$

and, due to $(3.3),\|\cdot\|_{\mathbf{d}}$ is continuous at zero.

Proposition 3.5. [38] If $\boldsymbol{d}$ is a strictly monotone dilation then :

- the canonical homogeneous norm $\|\cdot\|_{d}$ is Lipschitz continuous on $\mathbb{R}^{n} \backslash\{\mathbf{0}\}$;

- if the norm $\|\cdot\|$ is smooth outside the origin then the homogeneous norm $\|\cdot\|_{d}$ is also smooth outside the origin, $\frac{d\|\boldsymbol{d}(-s) x\|}{d s}<0$ if $s \in \mathbb{R}, x \in \mathbb{R}^{n} \backslash\{\mathbf{0}\}$ and

$$
\frac{\partial\|x\|_{d}}{\partial x}=\left.\frac{\left.\|x\|_{d} \frac{\partial\|z\|}{\partial z}\right|_{z=d(-s) x}}{\left.\frac{\partial\|z\|}{\partial z}\right|_{z=d(-s) x} G_{d} d(-s) x}\right|_{s=\ln \|x\|_{d}}
$$


Below we use the notation $\|\cdot\|_{\mathbf{d}}$ only for the canonical homogeneous norm induced by the weighted Euclidean norm $\|x\|=\sqrt{x^{\top} P x}$ with a matrix $P \succ 0$ satisfying (3.2). The unit sphere $S$ is defined using the same norm.

Vector fields, which are homogeneous with respect to dilation $\mathbf{d}$, have many properties useful for control design and state estimation of linear and nonlinear plants as well as for analysis of convergence rates [21], [47], [39], [32].

Definition 3.6. [38] A vector field $f: \mathbb{R}^{n} \rightarrow \mathbb{R}^{n}$ (resp. a function $h: \mathbb{R}^{n} \rightarrow \mathbb{R}$ ) is said to be $\boldsymbol{d}$-homogeneous if there exists $\nu \in \mathbb{R}$

$$
\begin{aligned}
& f(\boldsymbol{d}(s) x)=e^{\nu s} \boldsymbol{d}(s) f(x), \forall x \in \mathbb{R}^{n} \backslash\{\mathbf{0}\}, \forall s \in \mathbb{R} . \\
& \left(\text { resp. } h(\boldsymbol{d}(s) x)=e^{\nu s} h(x), \forall x \in \mathbb{R}^{n} \backslash\{\mathbf{0}\}, \forall s \in \mathbb{R} .\right)
\end{aligned}
$$

The number $\nu \in \mathbb{R}$ is called the homogeneity degree of $f$ (resp. $h$ ).

Let $\mathbb{F}_{\boldsymbol{d}}\left(\mathbb{R}^{n}\right)$ (resp. $\mathbb{H}_{\boldsymbol{d}}\left(\mathbb{R}^{n}\right)$ ) be the set of vector fields $\mathbb{R}^{n} \rightarrow \mathbb{R}^{n}$ (resp. functions $\mathbb{R}^{n} \rightarrow \mathbb{R}$ ) satisfying the identity (3.6) (resp. (3.7)), which are continuous on $\mathbb{R}^{n} \backslash\{\mathbf{0}\}$. Let $\operatorname{deg}_{\mathbb{F}_{d}}(f)$ (resp. $\operatorname{deg}_{\mathbb{H}_{\boldsymbol{d}}}(h)$ ) denote the homogeneity degree of $f \in \mathbb{F}_{\boldsymbol{d}}\left(\mathbb{R}^{n}\right)$ (resp. $h \in \mathbb{H}_{\boldsymbol{d}}\left(\mathbb{R}^{n}\right)$ ).

The homogeneity allows local properties (e.g., smoothness) of vector fields (functions) to be extended globally [19], [20]. For instance [38], the vector field $f \in \mathbb{F}_{\mathbf{d}}\left(\mathbb{R}^{n}\right)$ is Lipschitz continuous on $\mathbb{R}^{n} \backslash\{\mathbf{0}\}$ if and only if it satisfies Lipschitz condition on the unit sphere $S$. The next proposition uses similar arguments to specify uniform continuity property of homogeneous vector fields. Since the map $s \rightarrow \mathbf{d}(s) x$ is locally uniformly continuous, then uniform continuity of $f \in \mathbb{F}_{\mathbf{d}}\left(\mathbb{R}^{n}\right)$ on the unit sphere implies its local uniform continuity on $\mathbb{R}^{n} \backslash\{\mathbf{0}\}$.

Proposition 3.7. If $f \in \mathbb{F}_{\boldsymbol{d}}\left(\mathbb{R}^{n}\right)$ is uniformly continuous ${ }^{2}$ on the unit sphere $S$ with the modulus of continuity $\omega \in \mathcal{K}$, then it is uniformly continuous on any set

$$
K\left(r_{1}, r_{2}\right)=\left\{x \in \mathbb{R}^{n}: r_{1} \leq\|x\|_{\boldsymbol{d}} \leq r_{2}\right\}, \quad 0<r_{1}<1<r_{2}
$$

with the modulus of continuity

$$
\omega_{r_{1}, r_{2}}(\sigma) \leq \max \left\{r_{1}^{\nu}, r_{2}^{\nu}\right\}\left(r_{1}^{\beta-\alpha}+r_{2}^{\alpha-\beta}\right)\left(\frac{r_{2}^{\alpha}}{r_{1}^{\beta-\alpha}+r_{2}^{\alpha-\beta}} \omega\left(\frac{M\left(r_{1}^{\beta-\alpha}+r_{2}^{\alpha-\beta}\right) \sigma}{r_{1}^{\alpha}}\right)+M \sigma\right)
$$

where $\nu=\operatorname{deg}_{\mathbb{F}_{d}}(f), 0<\beta \leq \alpha$ are defined in Theorem 3.3 and $M>0$ is a constant number independent of $r_{1}$ and $r_{2}$.

Proof. I. If $u_{1}, u_{2} \in K\left(r_{1}, r_{2}\right)$ and $\left\|u_{1}\right\|_{\mathbf{d}} \geq\left\|u_{2}\right\|_{\mathbf{d}}$ then

$$
\begin{gathered}
1=\left\|\mathbf{d}\left(-\ln \left\|u_{1}\right\|_{\mathbf{d}}\right) u_{1}\right\|=\left\|\mathbf{d}\left(-\ln \left\|u_{1}\right\|_{\mathbf{d}}\right)\left(u_{1}-u_{2}\right)+\mathbf{d}\left(\ln \frac{\left\|u_{2}\right\|_{\mathbf{d}}}{\left\|u_{1}\right\|_{\mathbf{d}}}\right) \mathbf{d}\left(-\ln \left\|u_{2}\right\|_{\mathbf{d}}\right) u_{2}\right\| \leq \\
\left\|\mathbf{d}\left(\ln \frac{r_{1}}{\left\|u_{1}\right\|_{\mathbf{d}}}\right)\right\|\left\|\mathbf{d}\left(-\ln r_{1}\right)\right\|\left\|\left(u_{1}-u_{2}\right)\right\|+\left\|\mathbf{d}\left(\ln \frac{\left\|u_{2}\right\|_{\mathbf{d}}}{\left\|u_{1}\right\|_{\mathbf{d}}}\right)\right\| .
\end{gathered}
$$

\footnotetext{
${ }^{2} \mathrm{~A}$ map $f: \mathbb{R}^{n} \rightarrow \mathbb{R}^{n}$ is said to be uniformly continuous on a set $\Omega$ if there exists $\omega \in \mathcal{K}$ such that $\left\|f\left(x_{1}\right)-f\left(x_{2}\right)\right\| \leq \omega\left(\left\|x_{1}-x_{2}\right\|\right)$ for any $x_{1}, x_{2} \in \Omega$, where the function $\omega$ is the so-called modulus of continuity.
} 
From Theorem 3.3 we infer:

$$
\left\|\mathbf{d}\left(\ln \frac{\left\|u_{2}\right\|_{\mathbf{d}}}{\left\|u_{1}\right\|_{\mathbf{d}}}\right)\right\| \leq e^{\beta \ln \frac{\left\|u_{2}\right\|_{\mathbf{d}}}{\left\|u_{1}\right\|_{\mathbf{d}}}},\left\|\mathbf{d}\left(\ln \frac{r_{1}}{\left\|u_{1}\right\|_{\mathbf{d}}}\right)\right\| \leq e^{\beta \ln \frac{r_{1}}{\left\|u_{1}\right\|_{\mathbf{d}}}}
$$

and $\left\|\mathbf{d}\left(-\ln r_{1}\right)\right\| \leq e^{-\alpha \ln r_{1}}$. Thus we have

$$
\left|\left\|u_{1}\right\|_{\mathbf{d}}^{\beta}-\left\|u_{2}\right\|_{\mathbf{d}}^{\beta}\right| \leq r_{1}^{\beta-\alpha}\left\|u_{1}-u_{2}\right\|
$$

Similarly we prove that

$$
\left|\left\|u_{1}\right\|_{\mathbf{d}}^{\alpha}-\left\|u_{2}\right\|_{\mathbf{d}}^{\alpha}\right| \leq r_{2}^{\alpha-\beta}\left\|u_{1}-u_{2}\right\| .
$$

II. Since

$$
\left\|\int_{s_{2}}^{s_{1}} \mathbf{d}(s) d s\right\| \leq \frac{\left\|G_{\mathbf{d}}\right\|}{\beta}\left|e^{\beta s_{1}}-e^{\beta s_{2}}\right| \text { for } s_{1}, s_{2} \geq 0
$$

and

$$
\left\|\int_{s_{2}}^{s_{1}} \mathbf{d}(s) d s\right\| \leq \frac{\left\|G_{\mathbf{d}}\right\|}{\alpha}\left|e^{\alpha s_{1}}-e^{\alpha s_{2}}\right| \text { for } s_{1}, s_{2} \leq 0
$$

then taking into account $\alpha \geq \beta$ we conclude

$$
\left\|\int_{s_{2}}^{s_{1}} \mathbf{d}(s) d s\right\| \leq \frac{\left|e^{\alpha s_{1}}-e^{\alpha s_{2}}\right|+\left|e^{\beta s_{1}}-e^{\beta s_{2}}\right|}{\beta} \text { for } s_{1}, s_{2} \in \mathbb{R} .
$$

Similarly we derive the following inequality

$$
\left\|\int_{s_{2}}^{s_{1}} e^{\nu s} \mathbf{d}(s) d s\right\| \leqslant e^{\max \left\{\nu s_{1}, \nu s_{2}\right\}} \frac{\left|e^{\alpha s_{1}}-e^{\alpha s_{2}}\right|+\left|e^{\beta s_{1}}-e^{\beta s_{2}}\right|}{\beta} .
$$

III. Let us denote $z_{i}=\mathbf{d}\left(-\ln \left\|u_{i}\right\|_{\mathbf{d}}\right) u_{i} \in S, i=1,2$. Then

$$
\begin{gathered}
f\left(u_{1}\right)-f\left(u_{2}\right)=f\left(\mathbf{d}\left(\ln \left\|u_{1}\right\|_{\mathbf{d}}\right) z_{1}\right)-f\left(\mathbf{d}\left(\ln \left\|u_{2}\right\|_{\mathbf{d}}\right) z_{2}\right) \\
=\left\|u_{1}\right\|_{\mathbf{d}}^{\nu} \mathbf{d}\left(\ln \left\|u_{1}\right\|_{\mathbf{d}}\right) f\left(z_{1}\right)-\left\|u_{2}\right\|_{\mathbf{d}}^{\nu} \mathbf{d}\left(\ln \left\|u_{2}\right\|_{\mathbf{d}}\right) f\left(z_{2}\right) \\
=\left\|u_{1}\right\|_{\mathbf{d}}^{\nu} \mathbf{d}\left(\ln \left\|u_{1}\right\|_{\mathbf{d}}\right)\left(f\left(z_{1}\right)-f\left(z_{2}\right)\right)+\left(\left\|u_{1}\right\|_{\mathbf{d}}^{\nu} \mathbf{d}\left(\ln \left\|u_{1}\right\|_{\mathbf{d}}\right)-\left\|u_{2}\right\|^{\nu} \mathbf{d}\left(\ln \left\|u_{2}\right\|_{\mathbf{d}}\right)\right) f\left(z_{2}\right) .
\end{gathered}
$$

Since $f$ is uniformly continuous on $S$ then

$$
\left\|f\left(u_{1}\right)-f\left(u_{2}\right)\right\| \leq \max \left\{r_{1}^{\nu}, r_{2}^{\nu}\right\} r_{2}^{\alpha} \omega\left(\left\|z_{1}-z_{2}\right\|\right)+f^{\max }\left\|e^{\left(\nu I_{n}+G_{\mathbf{d}}\right) \ln \left\|u_{1}\right\|_{\mathbf{d}}}-e^{\left(\nu I_{n}+G_{\mathbf{d}}\right) \ln \left\|u_{2}\right\|_{\mathbf{d}}}\right\|,
$$


where $f^{\max }=\sup _{x \in S}\|f(x)\|$. Finally, it follows that

$$
\begin{gathered}
\left\|z_{1}-z_{2}\right\|=\left\|\mathbf{d}\left(-\ln \left\|u_{1}\right\|_{\mathbf{d}}\right) u_{1}-\mathbf{d}\left(-\ln \left\|u_{2}\right\|_{\mathbf{d}}\right) u_{2}\right\|= \\
\left\|\mathbf{d}\left(\ln \frac{1}{\left\|u_{1}\right\|_{\mathbf{d}}}\right)\left(u_{1}-u_{2}+\left(\mathbf{d}\left(\ln \left\|u_{2}\right\|_{\mathbf{d}}\right)-\mathbf{d}\left(\ln \left\|u_{1}\right\|_{\mathbf{d}}\right)\right) z_{2}\right)\right\| \leq \\
\left\|\mathbf{d}\left(\ln \frac{1}{\left\|u_{1}\right\|_{\mathbf{d}}}\right)\right\|\left(\left\|u_{1}-u_{2}\right\|+\left\|G_{\mathbf{d}} \int_{\ln \left\|u_{2}\right\|}^{\ln \left\|u_{1}\right\|} \mathbf{d}(s) d s\right\|\right) \leq \frac{1+\beta^{-1}\left\|G_{\mathbf{d}}\right\|\left(r_{1}^{\beta-\alpha}+r_{2}^{\alpha-\beta}\right)}{r_{1}^{\alpha}}\left\|u_{1}-u_{2}\right\|,
\end{gathered}
$$

and

$$
\begin{gathered}
\|\| u_{1}\left\|_{\mathbf{d}}^{\nu} \mathbf{d}\left(\ln \left\|u_{1}\right\|_{\mathbf{d}}\right)-\right\| u_{2}\left\|^{\nu} \mathbf{d}\left(\ln \left\|u_{2}\right\|_{\mathbf{d}}\right)\right\|=\left\|\left(\nu I_{n}+G_{\mathbf{d}}\right) \int_{\ln \left\|u_{2}\right\|}^{\ln \left\|u_{1}\right\|} e^{\nu s} \mathbf{d}(s) d s\right\| \leq \\
\frac{\left\|\nu I_{n}+G_{\mathbf{d}}\right\| \max \left\{\left\|u_{1}\right\|_{\mathbf{d}}^{\nu},\left\|u_{2}\right\|_{\mathbf{d}}^{\nu}\right\}\left(\left|\left\|u_{1}\right\|_{\mathbf{d}}^{\alpha}-\left\|u_{2}\right\|_{\mathbf{d}}^{\alpha}\right|+\left|\left\|u_{1}\right\|_{\mathbf{d}}^{\beta}-\left\|u_{2}\right\|_{\mathbf{d}}^{\beta}\right|\right)}{\beta} \leq \\
\frac{\left\|\nu I_{n}+G_{\mathbf{d}}\right\| \max \left\{r_{1}^{\nu}, r_{2}^{\nu}\right\}\left(r_{1}^{\beta-\alpha}+r_{2}^{\alpha-\beta}\right)}{\beta}\left\|u_{1}-u_{2}\right\|
\end{gathered}
$$

3.2. Quadratic Stability of Nonlinear Homogeneous Systems. Homogeneity may simplify the analysis of differential equations. The most important property of $\mathbf{d}$-homogeneous systems is the symmetry of solutions [19], [30], [31],[22], [39]. Namely, if $\varphi_{\xi_{0}}:[0, T) \rightarrow \mathbb{R}^{n}$ is a solution to

$$
\dot{\xi}=f(\xi), \quad f \in \mathbb{F}_{\boldsymbol{d}}\left(\mathbb{R}^{n}\right)
$$

with the initial condition $\xi(0)=\xi_{0} \in \mathbb{R}^{n}$, then $\varphi_{\boldsymbol{d}(s) \xi_{0}}:\left[0, e^{-\nu s} T\right) \rightarrow \mathbb{R}^{n}$ defined as

$$
\varphi_{\boldsymbol{d}(s) \xi_{0}}(t)=\boldsymbol{d}(s) \varphi_{\xi_{0}}\left(t e^{\nu s}\right), \quad s \in \mathbb{R}
$$

is a solution to (3.8) with the initial condition $\xi(0)=\boldsymbol{d}(s) \xi_{0}$, where $\nu=\operatorname{deg}_{\mathbb{F}_{d}}(f)$.

The latter property implies many corollaries. In this paper we use the next one.

Theorem 3.8. [38] The next five claims are equivalent:

1) The origin of the system (3.8) is asymptotically stable.

2) There exists a Lyapunov function $V$ for the system (3.8) such that

$$
V \in \mathbb{H}_{\boldsymbol{d}}\left(\mathbb{R}^{n}\right) \cap C^{\infty}\left(\mathbb{R}^{n}\right) .
$$

3) The origin of the system

$$
\dot{z}=\|z\|^{1+\operatorname{deg}_{\mathbb{F}_{d}}(f)}\left(\frac{\left(I_{n}-G_{d}\right) z z^{\top} P}{z^{\top} P G_{d} z}+I_{n}\right) f\left(\frac{z}{\|z\|}\right)
$$

is asymptotically stable, where $z=\sqrt{z^{\top} P z}$, and the positive definite matrix $P \in \mathbb{R}^{n \times n}$ satisfies (3.2). 
4) For any positive definite matrix $P \in \mathbb{R}^{n \times n}$ satisfying (3.2) there exists

$$
\Psi \in \mathbb{F}_{\boldsymbol{d}}\left(\mathbb{R}^{n}\right) \cap C^{\infty}\left(\mathbb{R}^{n} \backslash\{\mathbf{0}\}\right), \quad \operatorname{deg}_{\mathbb{F}_{d}}(\Psi)=0,
$$

such that $\Psi$ is a diffeomorphism on $\mathbb{R}^{n} \backslash\{\mathbf{0}\}$, a homeomorphism on $\mathbb{R}^{n}, \Psi(\mathbf{0})=\mathbf{0}$ and

$$
\frac{\partial\left(\Psi^{\top}(\xi) P \Psi(\xi)\right)}{\partial \xi} f(\xi)<0 \quad \text { if } \Psi^{\top}(\xi) P \Psi(\xi)=1 .
$$

Moreover, $\|\Psi\|_{\boldsymbol{d}} \in \mathbb{H}_{\boldsymbol{d}}\left(\mathbb{R}^{n}\right) \cap C^{\infty}\left(\mathbb{R}^{n} \backslash\{\mathbf{0}\}\right)$ is a Lyapunov function for the system (3.8).

5) For any matrix $P \in \mathbb{R}^{n \times n}$ satisfying (3.2), there exists a smooth mapping

$$
\Xi \in C^{\infty}\left(\mathbb{R}^{n} \backslash\{\mathbf{0}\}, \mathbb{R}^{n \times n}\right)
$$

such that

$$
\begin{gathered}
\operatorname{det}(\Xi(z)) \neq 0, \quad \frac{\partial \Xi(z)}{\partial z_{i}} z=0, \quad \Xi\left(e^{s} z\right)=\Xi(z) \\
\text { for } z=\left(z_{1}, \ldots, z_{n}\right)^{\top} \in \mathbb{R}^{n} \backslash\{\mathbf{0}\}, s \in \mathbb{R}, i=1, \ldots, n
\end{gathered}
$$

and

$$
z^{\top} \Xi^{\top}(z) P \Xi(z)\left(\frac{\left(I_{n}-G_{d}\right) z z^{\top} P}{z^{\top} P G_{d} z}+I_{n}\right) f\left(\frac{z}{\|z\|}\right)<0
$$

This theorem proves two important facts:

- Any generalized homogeneous system (3.8) is homeomorphic on $\mathbb{R}^{n}$ and diffeomorphic on $\mathbb{R}^{n} \backslash\{\mathbf{0}\}$ to a standard homogeneous one (3.9). The corresponding change of coordinates is given by

$$
z=\|\xi\|_{\mathbf{d}} \mathbf{d}\left(-\ln \|\xi\|_{\mathbf{d}}\right) \xi
$$

while the inverse transformation is as follows:

$$
\xi=\mathbf{d}(\ln \|z\|) \frac{z}{\|z\|}
$$

- Any asymptotically stable generalized homogeneous system is homeomorphic on $\mathbb{R}^{n}$ and diffeomorphic on $\mathbb{R}^{n} \backslash\{\mathbf{0}\}$ to a quadratically stable one. Indeed, making the change of variables $z=\Psi(\xi)$ we derive

$$
\dot{z}=f_{0}(z)=\left.\frac{\partial \Psi(\xi)}{\partial \xi} f(\xi)\right|_{\xi=\Psi^{-1}(z)},
$$

but the criterion (3.10) implies that $z^{\top} P \dot{z}<0$ if $z^{\top} P z=1$, so the homogeneous norm $\|\cdot\|_{\mathbf{d}}$ is the Lyapunov function to the latter system. Finally, the change of variable $x=\|z\|_{\mathbf{d}} \mathbf{d}\left(-\ln \|z\|_{\mathbf{d}}\right) z$ gives $\|z\|_{\mathbf{d}}=\|x\|$, so the transformed system $\dot{x}=\tilde{f}(x)$ is quadratically stable with the Lyapunov function $V$ defined as $V(x)=\|x\|^{2}=x^{\top} P x$, where

$$
\tilde{f}(x)=\|x\|^{1+\operatorname{deg}_{\mathbb{F}_{\mathbf{d}}}(f)}\left(\frac{\left(I_{n}-G_{\mathbf{d}}\right) x x^{\top} P}{x^{\top} P G_{\mathbf{d}} x}+I_{n}\right) f_{0}\left(\frac{x}{\|x\|}\right) .
$$


Lemma 3.9. If a $\boldsymbol{d}$-homogeneous vector field $f \in \mathbb{F}_{\boldsymbol{d}}\left(\mathbb{R}^{n}\right)$ is uniformly continuous on $S$, then for any positive definite matrix $P \in \mathbb{R}^{n \times n}$ satisfying (3.2) the vector field $\tilde{f}: \mathbb{R}^{n} \rightarrow \mathbb{R}^{n}$ defined as

$$
\tilde{f}(z)=\left(z^{\top} P z\right)^{0.5\left(1+\operatorname{deg}_{\mathbb{F}_{d}}(f)\right)}\left(\frac{\left(I_{n}-G_{d}\right) z^{\top} z P}{z^{\top} P G_{d} z}+I_{n}\right) f\left(\frac{z}{\sqrt{z^{\top} P z}}\right)
$$

is uniformly continuous on $S$.

Proof. Let us rewrite $\tilde{f}(z)$ as follows

$$
\tilde{f}(z)=\|z\|^{1+\operatorname{deg}_{\mathbb{F}_{\mathbf{d}}}(f)}\left(I_{n}-G_{\mathbf{d}}\right) \frac{z^{\top} P f\left(\frac{z}{\|z\|}\right)}{z^{\top} P G_{\mathbf{d}} z} z+\|z\|^{1+\operatorname{deg}_{\mathbb{F}_{\mathbf{d}}}(f)} f\left(\frac{z}{\|z\|}\right) .
$$

By assumption of the proposition, the second term defines a uniformly continuous function on $S$. To complete the proof we need to study of the first term only. If $z_{1}, z_{2} \in S$ then

$0<\beta \leq z_{i}^{\top} P G_{\mathbf{d}} z_{i} \leq \alpha, i=1,2 \quad$ and $\quad\left\|\frac{z_{1}^{\top} P f\left(z_{1}\right)}{z_{1}^{\top} P G_{\mathbf{d}} z_{1}} z_{1}-\frac{z_{2}^{\top} P f\left(z_{2}\right)}{z_{2}^{\top} P G_{\mathbf{d}} z_{2}} z_{2}\right\| \leq \frac{\left\|q\left(z_{1}\right) p\left(z_{2}\right) z_{1}-q\left(z_{2}\right) p\left(z_{1}\right) z_{2}\right\|}{\beta^{2}}$,

where $0<\beta \leq \alpha$ are defined in Theorem 3.3 and $q(a)=a^{\top} P f(a), p(b)=b^{\top} P G_{\mathbf{d}} b$ with $a, b \in \mathbb{R}^{n}$. We have

$$
\begin{gathered}
\left\|q\left(z_{1}\right) p\left(z_{2}\right) z_{1}-q\left(z_{2}\right) p\left(z_{1}\right) z_{2}\right\| \leq \\
\left\|q\left(z_{1}\right) p\left(z_{2}\right) z_{1}-q\left(z_{1}\right) p\left(z_{2}\right) z_{2}+q\left(z_{1}\right) p\left(z_{2}\right) z_{2}-q\left(z_{2}\right) p\left(z_{1}\right) z_{2}\right\| \leq \\
\left\|q\left(z_{1}\right) p\left(z_{2}\right)\right\| \cdot\left\|z_{1}-z_{2}\right\|+ \\
\left\|q\left(z_{1}\right) p\left(z_{2}\right)-q\left(z_{1}\right) p\left(z_{1}\right)+q\left(z_{1}\right) p\left(z_{1}\right)-q\left(z_{2}\right) p\left(z_{1}\right)\right\| \leq \\
\alpha k\left\|z_{1}-z_{2}\right\|+k\left\|p\left(z_{2}\right)-p\left(z_{1}\right)\right\|+\alpha\left\|q\left(z_{1}\right)-q\left(z_{2}\right)\right\|,
\end{gathered}
$$

where $k=\sup _{z \in S} z^{\top} P f(z)$. The function $p$ is continuously differentiable and positive definite. Since $f$ is uniformly continuous on $S$ then $q$ is uniformly continuous on $S$ and, consequently, $\tilde{f}$ is uniformly continuous on $S$.

Recall [28], [29], [39], [38] that if the standard (or weighted/generalized) homogeneous system (3.8) is asymptotically stable and $\operatorname{deg}_{\mathbb{F}_{\mathbf{d}}}(f)<0$, then it is globally uniformly finitetime stable.

Remark 3.10. If $\boldsymbol{d}$ is a dilation with the generator $G_{\boldsymbol{d}}$, then for any fixed $\alpha>0$, the group $\boldsymbol{d}^{\alpha}$ defined as $\boldsymbol{d}^{\alpha}(s):=\boldsymbol{d}(\alpha s), s \in \mathbb{R}^{n}$, is the dilation with the generator $G_{\boldsymbol{d}^{\alpha}}=\alpha G_{\boldsymbol{d}}$. Moreover, if $f \in \mathbb{F}_{\boldsymbol{d}}\left(\mathbb{R}^{n}\right)$, then $f \in \mathbb{F}_{\boldsymbol{d}^{\alpha}}\left(\mathbb{R}^{n}\right)$ and $\operatorname{deg}_{\mathbb{F}_{d^{\alpha}}}(f)=\alpha \operatorname{deg}_{\mathbb{F}_{d}}(f)$. In other words, if $\operatorname{deg}_{\mathbb{F}_{d}}(f)<0$, then a new dilation $\boldsymbol{d}^{\alpha}$ can be selected such that $\operatorname{deg}_{\mathbb{F}_{d^{\alpha}}}(f)=-1$. Similarly, if $\operatorname{deg}_{\mathbb{F}_{\boldsymbol{d}}}(f)>0$, then a new dilation $\boldsymbol{d}^{\alpha}$ can be selected such that $\operatorname{deg}_{\mathbb{F}_{d^{\alpha}}}(f)=1$.

4. Consistent Implicit Discretization of Finite-time Stable Homogeneous Systems. The main idea of the design of finite-time stable discretization for homogeneous systems, is to use the coordinate transformation (3.12). If $\operatorname{deg}_{\mathbb{F}}(f)=-1$, then the right-hand side of the transformed system (3.9) is globally bounded. Notice that for the scalar system studied in the introduction, the transformation (3.12) is $y=\sqrt{|x|} \operatorname{sgn}(x)$ with the inverse $x=y^{2} \operatorname{sgn}(y)$. 
Theorem 4.1. Let a d-homogeneous vector field $f \in \mathbb{F}_{\boldsymbol{d}}\left(\mathbb{R}^{n}\right)$ be uniformly continuous on $S$, $\operatorname{deg}_{\mathbb{F}_{d}}(f)=-1, f(-x)=-f(x)$ for $x \in \mathbb{R}^{n} \backslash\{\mathbf{0}\}$ and

$$
\tilde{f}(y):=\left(\frac{\left(I-G_{d}\right) y y^{\top} P}{y^{\top} G_{d} P y}+I_{n}\right) f\left(\frac{y}{\sqrt{y^{\top} P y}}\right), \quad y \in \mathbb{R}^{n} \backslash\{\mathbf{0}\},
$$

where $G_{\boldsymbol{d}}$ is the generator of the dilation $\boldsymbol{d}$ and the symmetric matrix $P \in \mathbb{R}^{n \times n}$ satisfies (3.2). If the condition (3.11) holds with $\Xi=I_{n}$, then the map $Q: \mathbb{R}_{+} \times \mathbb{R}^{n} \times \mathbb{R}^{n} \rightrightarrows \mathbb{R}^{n}$ defined as

$$
\begin{gathered}
Q\left(h, x_{i}, x_{i+1}\right)=\tilde{Q}\left(h, \Phi\left(x_{i}\right), \Phi\left(x_{i+1}\right)\right), \\
\Phi(x)=\|x\|_{\boldsymbol{d}} \boldsymbol{d}\left(-\ln \|x\|_{\boldsymbol{d}}\right) x
\end{gathered}
$$

where $h>0$ and

$$
\begin{gathered}
\tilde{Q}\left(h, y_{i}, y_{i+1}\right)=y_{i+1}-y_{i}-h \tilde{F}\left(y_{i+1}\right), \\
\tilde{F}(y)=\bigcap_{\varepsilon>0} \operatorname{co} \tilde{f}(y+\varepsilon \mathcal{B} \backslash\{\mathbf{0}\}),
\end{gathered}
$$

is a consistent discrete-time approximation of the finite-time stable system (2.1).

Proof. First of all, let us note that the system

$$
\dot{y}=\tilde{f}(y),
$$

is equivalent to the system (2.1) with the change of coordinates

$$
y=\Phi(x), \quad x=\Phi^{-1}(y)=\mathbf{d}(\ln \|y\|) \frac{y}{\|y\|} .
$$

For more details about the coordinate transformation $\Phi$ we refer the reader to [37], [38] and/or to explanations presented after Theorem 3.8 given above. If we show that $\tilde{Q}$ is a discrete-time approximation of (4.3) then, due to continuity of $\Phi$ on $\mathbb{R}^{n}$ and its smoothness on $\mathbb{R}^{n} \backslash\{\mathbf{0}\}$, we would derive that $Q$ is a discrete-time approximation of (2.1).

Note also that by construction $\tilde{f}$ is globally bounded and continuous on $\mathbb{R}^{n} \backslash\{\mathbf{0}\}$, and $\tilde{f}(\alpha y)=\tilde{f}(y)$ for any $\alpha>0$ and any $y \in \mathbb{R}^{n} \backslash\{\mathbf{0}\}$.

1) Existence property. Let us show that for any $\tilde{y} \in \mathbb{R}^{n}$ and any $h>0$, there exists $\tilde{y}_{h} \in \mathbb{R}^{n}$ such that $0 \in \tilde{Q}\left(h, \tilde{y}, \tilde{y}_{h}\right)$. By construction, $\tilde{F}(\mathbf{0})$ is non-empty, convex and compact set. In addition, since $f$ is central symmetric, i.e., $f(-x)=-f(x)$, then $\tilde{f}$ is also central symmetric with $\tilde{f}(-y)=-\tilde{f}(y)$, and $\tilde{y} \in \tilde{F}(\mathbf{0}) \Rightarrow-\tilde{y} \in \tilde{F}(\mathbf{0})$. The latter implies that the set $\tilde{F}(\mathbf{0})$ is symmetric and $\mathbf{0} \in \tilde{F}(\mathbf{0})$ due to convexity of $\tilde{F}(\mathbf{0})$. Since $\tilde{f}\left(e^{s} y\right)=\tilde{f}(y)$ for $y \in \mathbb{R}^{n} \backslash\{\mathbf{0}\}$ and $s \in \mathbb{R}$ then $\tilde{f}(y) \in \tilde{F}(0)$ for any $y \in \mathbb{R}^{n}$ by construction.

Case $\tilde{y} \in \Omega:=h \tilde{F}(\mathbf{0})$. Obviously, if $\tilde{y} \in \Omega$ then for $\tilde{y}_{h}=\mathbf{0}$ one has $\mathbf{0} \in \tilde{Q}\left(h, \tilde{y}, \tilde{y}_{h}\right)$. Let us show that the inclusion $\mathbf{0} \in \tilde{Q}\left(h, \mathbf{0}, \tilde{y}_{h}\right)$ has the unique solution $\tilde{y}_{h}=\mathbf{0}$. Suppose the contrary, i.e., $\tilde{y}_{h} \neq \mathbf{0}$. Then the inclusion must hold as identity $\tilde{y}_{h}=h \tilde{f}\left(\tilde{y}_{h}\right)$. Hence, $0<\tilde{y}_{h}^{\top} \Xi P \Xi \tilde{y}_{h}=h \tilde{y}_{h}^{\top} \Xi P \Xi \tilde{f}\left(\tilde{y}_{h}\right)<0$ due to (3.11), i.e., we obtain the contradiction. 
Case $\tilde{y} \notin \Omega$. In this case, $\tilde{y}+\Delta \neq \mathbf{0}$ for any $\Delta \in \Omega$. Indeed, otherwise, $\Delta=-\tilde{y} \in \Omega$ and $\tilde{y} \in \Omega$ due to symmetry of $\tilde{F}(\mathbf{0})$. Therefore, for any $\Delta \in \Omega$ one has $\tilde{y}+\Delta \neq \mathbf{0}$ and $\tilde{F}(\tilde{y}+\Delta)=\tilde{f}(\tilde{y}+\Delta)$. Moreover, $h \tilde{f}(\tilde{y}+\cdot): \Omega \rightarrow \Omega$ is continuous. Since $\Omega$ is convex and compact, then according the Brouwer fixed-point theorem there exists $\Delta^{*} \in \Omega$ such that $\Delta^{*}=h \tilde{f}\left(\tilde{y}+\Delta^{*}\right)$ and taking $\tilde{y}_{h}=\tilde{y}+\Delta^{*}$ we derive $\tilde{Q}\left(h, \tilde{y}, \tilde{y}_{h}\right)=0$.

2) Finite-time convergence property. Let us show that the sequence $\left\{y_{i}\right\}_{i=0}^{+\infty}$ generated by $0 \in \tilde{Q}\left(h, y_{i}, y_{i+1}\right)$ converges to zero in a finite number of steps. Since the stability criterion (3.11) holds for $\Xi=I_{n}$, then $V(z)=z^{\top} P z$ is a Lyapunov function for the system (4.3). Let us show that there exists $i^{*}$ such that $y_{i^{*}}=0$. Suppose the contrary, i.e., $y_{i} \neq 0$ for all $i>0$. In this case one has $y_{i-1}=y_{i}-h \tilde{f}\left(y_{i}\right)$ and

$$
\begin{gathered}
V\left(y_{i-1}\right)=\left(y_{i}-\tilde{f}\left(y_{i}\right)\right)^{\top} P\left(y_{i}-h \tilde{f}\left(y_{i}\right)\right)= \\
V\left(y_{i}\right)-2 h y_{i}^{\top} P \tilde{f}\left(y_{i}\right)+h^{2} \tilde{f}^{\top}\left(y_{i}\right) P \tilde{f}\left(y_{i}\right)>V\left(y_{i}\right)+c,
\end{gathered}
$$

where $c=h^{2} \inf _{z \in S} \tilde{f}^{\top}(z) P \tilde{f}(z)>0$. This means that in a finite number of steps we will have $V\left(y_{i}\right)<0$, but this is impossible due to positive definiteness of the Lyapunov function $V$. Therefore, there exists $i^{*}$ such that $y_{i^{*}}=0$.

3) Approximation property. In view of Lemma 3.9, $\tilde{f}$ is uniformly continuous on $S$.

Let $0<r_{1}<1<r_{2}$ be arbitrary numbers and $y_{i}, y_{i+1} \in K\left(r_{1}, r_{2}\right)$, where the set $K\left(r_{1}, r_{2}\right)$ is defined in Proposition 3.7. According to Proposition 3.7 the function $\tilde{f}$ is uniformly continuous on $K\left(r_{1}, r_{2}\right)$, so

$$
\begin{gathered}
\left\|\tilde{\phi}\left(h, y_{i}\right)-y_{i+1}\right\|=\left\|\int_{0}^{h} \tilde{f}\left(\tilde{\phi}\left(\tau, y_{i}\right)\right) d \tau-h \tilde{f}\left(y_{i+1}\right)\right\|= \\
\left\|\int_{0}^{h} \tilde{f}\left(y_{i}+\int_{0}^{\tau} \tilde{f}\left(\tilde{\phi}\left(\sigma, y_{i}\right)\right) d \sigma\right)-\tilde{f}\left(y_{i}+h \tilde{f}\left(y_{i+1}\right)\right) d \tau\right\| \leq \\
h \sup _{\tau \in[0, h]} \omega_{r_{1}, r_{2}}\left(\left\|\int_{0}^{\tau} \tilde{f}\left(\tilde{\phi}\left(\sigma, y_{i}\right)\right) d \sigma-h \tilde{f}\left(y_{i+1}\right)\right\|\right) \leq h \omega_{r_{1}, r_{2}}\left(2 f^{\max } h\right),
\end{gathered}
$$

where $f^{\max }=\sup _{y \in K\left(r_{1}, r_{2}\right)} \tilde{f}(y)$ and $\tilde{\phi}\left(\cdot, y_{i}\right)$ is a solution to (4.3) with $y(0)=y_{i}$.

Since $\Phi^{-1}$ is continuously differentiable on $\mathbb{R}^{n} \backslash\{\boldsymbol{0}\}$, it satisfies a Lipschitz condition on $K\left(r_{1}, r_{2}\right)$, with a Lipschitz constant $L=L\left(r_{1}, r_{2}\right)$. If $\phi\left(h, x_{i}\right)$ is a solution to (2.1) with $x(0)=x_{i}$ then

$$
\left.\left\|\phi\left(h, x_{i}\right)-x_{i+1}\right\|=\left\|\Phi^{-1}\left(\tilde{\phi}\left(h, y_{i}\right)\right)-\Phi^{-1}\left(y_{i+1}\right)\right\| \leq L \| \tilde{\phi}\left(h, y_{i}\right)\right)-y_{i+1} \| \leq h L \omega_{r_{1}, r_{2}}\left(2 f^{\max } h\right) .
$$

The latter theorem is based on the fact that the system $\dot{y}=\tilde{f}(y)$ admits a quadratic Lyapunov function (the condition (3.11) with $\Xi=$ const). However, as it was shown in Theorem 3.8 , any stable homogeneous system is equivalent to a quadratically stable one. If $f$ in Theorem 4.1 is replaced with the equivalent one:

$$
f^{n e w}(x)=\left.\frac{\partial \Psi(\xi)}{\partial \xi} f(\xi)\right|_{\xi=\Psi^{-1}(x)}, \quad x \in \mathbb{R}^{n}, \quad \xi \in \mathbb{R}^{n}
$$


where $\Psi \in \mathbb{F}_{\mathbf{d}}\left(\mathbb{R}^{n}\right)$ is a diffeomorphism on $\mathbb{R}^{n} \backslash\{\mathbf{0}\}$ given in Theorem 3.8, then the condition $\Xi=I_{n}$ required for Theorem 4.1 is fulfilled. Notice that the vector field $f^{n e w}$ is central symmetric provided that the vector fields $f$ and $\Psi$ are central symmetric too. Indeed, if $\Psi(-x)=-\Psi(x)$ then $\Psi^{-1}(-x)=-\Psi^{-1}(x), \frac{\partial \Psi(x)}{\partial x}=\left.\frac{\partial \Psi(y)}{\partial y}\right|_{y=-x}$ and, consequently, $f^{\text {new }}(-x)=-f^{\text {new }}(x)$.

According to Theorem 3.8 a homogeneous Lyapunov function $V \in \mathbb{H}_{\mathbf{d}}\left(\mathbb{R}^{n}\right) \cap C^{\infty}\left(\mathbb{R}^{n}\right)$ with degree $\operatorname{deg}_{\mathbb{H}}(V)=\mu>0$ can always be found for any asymptotically stable system with a homogeneous vector field $f \in \mathbb{F}_{\mathbf{d}}\left(\mathbb{R}^{n}\right)$. In this case, the required transformation $\Psi$ can be defined as follows

$$
\Psi(\xi)=\mathbf{d}\left(\ln \frac{V^{1 / \mu}(\xi)}{\|\xi\|_{\mathbf{d}}}\right) \xi
$$

(see [37] for more details). The Lyapunov function $V$ can always be selected symmetric, namely, $V(-x)=V(x)$. Indeed, if $\tilde{V}: \mathbb{R}^{n} \rightarrow[0,+\infty)$ is a Lyapunov function for a system with a central symmetric vector field $f(-x)=-f(x)$, then $V: \mathbb{R}^{n} \rightarrow[0,+\infty)$ given by $V(x)=\tilde{V}(x)+\tilde{V}(-x)$ is also a Lyapunov function for the same system:

$$
\dot{V}=\frac{\partial V(x)}{\partial x} f(x)+\left.\frac{\partial V(y)}{\partial y}\right|_{y=-x}(-f(x))<0 .
$$

Since $V(-x)=V(x)$ and $\|-x\|_{\mathbf{d}}=\|x\|_{\mathbf{d}}$ then $\Psi(-x)=-\Psi(x)$. Finally, taking into account Remark 3.10, we complete the proof of the following claim.

Theorem 4.2. Any central symmetric (i.e., satisfying $f(-x)=-f(x)$ ) generalized homogeneous finite-time stable system (2.1) with a possible discontinuity at the origin, admits a consistent implicit approximation.

In other words, if we know a homogeneous Lyapunov function for a finite-time stable homogeneous system then, we can easily design a consistent (in the sense of Definition 2.1) implicit discretization scheme.

The central symmetry of the vector field $f$ is utilized in Theorem 4.1 in order to prove the existence of solutions of the implicit discretization scheme (4.1). The symmetry helps us to check an applicability of the Brouwer fixed-point theorem. Possibly, this condition could be omitted in the case of the Kukatani fixed-point theorem [48]. This issue needs additional investigations and goes out of the scope of this paper.

5. Consistent Semi-Implicit Discretization of Practically Fixed-time Stable Homogeneous System. As it has been recalled in the introduction, a positive degree of homogeneity corresponds to the case of practical fixed-time stability of the origin. In view of Remark 3.10, we can restrict ourselves to the case $\operatorname{deg}_{\mathbb{F}_{\mathbf{d}}}(f)=1$ without loss of generality. Notice also that the homogeneous vector field with positive degree is always continuous at the origin (see e.g. the formula (3.9)).

Theorem 5.1. Let a d-homogeneous vector field $f \in \mathbb{F}_{\boldsymbol{d}}\left(\mathbb{R}^{n}\right)$ be uniformly continuous on $S$, $\operatorname{deg}_{\mathbb{F}_{d}}(f)=1$ and

$$
\tilde{f}(z)=\left(\frac{\left(I-G_{d}\right) z z^{\top} P}{z^{\top} G_{d} P z}+I_{n}\right) f\left(\frac{z}{\|z\|}\right), \quad z \in \mathbb{R}^{n} \backslash\{\mathbf{0}\}
$$


where $G_{\boldsymbol{d}}$ is the generator of the dilation $\boldsymbol{d},\|z\|=\sqrt{z^{\top} P z}$ and the positive definite matrix $P \in \mathbb{R}^{n \times n}$ satisfies (3.2). If the condition (3.11) holds with $\Xi=I_{n}$ and

$$
S \subset W_{\alpha}\left(\mathbb{R}^{n}\right) \quad \text { for any } \quad \alpha \in(0,+\infty),
$$

where $W_{\alpha}(z):=z-\alpha\|z\| \tilde{f}(z)$, then the function $q: \mathbb{R}_{+} \times \mathbb{R}^{n} \times \mathbb{R}^{n} \rightarrow \mathbb{R}^{n}$ defined as

$$
q\left(h, x_{i}, x_{i+1}\right)=\tilde{q}\left(h, \Phi\left(x_{i}\right), \Phi\left(x_{i+1}\right)\right),
$$

where $h>0, \Phi(x)=\|x\|_{d}, \boldsymbol{d}\left(-\ln \|x\|_{\boldsymbol{d}}\right) x$ for $x \in \mathbb{R}^{n}$, and

$$
\tilde{q}\left(h, y_{i}, y_{i+1}\right)=y_{i+1}-y_{i}-h\left\|y_{i}\right\|\left\|y_{i+1}\right\| \tilde{f}\left(y_{i+1}\right)
$$

is a consistent discrete-time approximation of the practically fixed-time stable system (2.1).

Proof. Notice that since $\operatorname{deg}_{\mathbb{F}_{\mathbf{d}}}(f)=1$, then the system

$$
\dot{y}=\|y\|^{2} \tilde{f}(y)
$$

is equivalent to the system (2.1) with the change of coordinates

$$
y=\Phi(x), \quad x=\Phi^{-1}(y)=\mathbf{d}(\ln \|y\|) \frac{y}{\|y\|} .
$$

For more details about the coordinate transformation $\Phi$ we refer the reader to [37], [38] and/or to explanations presented after Theorem 3.8 given above. Hence, $\tilde{q}$ in (5.2), (5.3) corresponds to the semi-implicit discretization

$$
\frac{y_{i+1}-y_{i}}{h}=\left\|y_{i}\right\|\left\|y_{i+1}\right\| \tilde{f}\left(y_{i+1}\right), \quad i=0,1,2, \ldots
$$

1) Existence Property. First of all, notice that for $y_{i}=\mathbf{0}$ the presented discretization scheme gives the unique solution $y_{i+1}=\mathbf{0}$. From the latter equation we derive

$$
\frac{y_{i+1}}{\left\|y_{i}\right\|}-h\left\|y_{i}\right\|\left\|\frac{y_{i+1}}{\left\|y_{i}\right\|}\right\| \tilde{f}\left(\frac{y_{i+1}}{\left\|y_{i}\right\|}\right)=\frac{y_{i}}{\left\|y_{i}\right\|},
$$

and using the condition (5.1) we conclude the existence of a solution to $\tilde{q}\left(h, y_{i}, y_{i+1}\right)=0$ for $\left\|y_{i}\right\| \neq 0$ and $h>0$.

2) Practical fixed-time convergence property. Let us show that any sequence $\left\{y_{i}\right\}_{i=0}^{+\infty}$ generated by $\tilde{q}\left(h, y_{i}, y_{i+1}\right)=0$ satisfies the practical fixed-time convergence property. Since the stability criterion (3.11) holds for $\Xi=I_{n}$, then

$$
V(z)=z^{\top} P z, \quad z \in \mathbb{R}^{n},
$$

defines a Lyapunov function for the system (5.4), so $y^{\top} P \tilde{f}(y)<0$ for $y \neq \mathbf{0}$, and we derive

$$
\begin{aligned}
& y_{i+1}^{\top} P y_{i+1}=y_{i+1}^{\top} P y_{i}+h\left\|y_{i}\right\|\left\|y_{i+1}\right\| y_{i+1}^{\top} P \tilde{f}\left(y_{i+1}\right) \\
& =y_{i+1}^{\top} P y_{i}+h\left\|y_{i}\right\|\left\|y_{i+1}\right\|^{2}\left(\frac{y_{i+1}}{\left\|y_{i+1}\right\|}\right)^{\top} P \tilde{f}\left(\frac{y_{i+1}}{\left\|y_{i+1}\right\|}\right)
\end{aligned}
$$




$$
\leq y_{i+1}^{\top} P y_{i}-\operatorname{ch}\left\|y_{i}\right\|\left\|y_{i+1}\right\|^{2}
$$

where

$$
c=\inf _{z \in S}-z^{\top} P \tilde{f}(z)>0 .
$$

Hence, using the Cauchy-Schwartz inequality we derive

$$
\left\|y_{i+1}\right\|^{2} \leq\left\|y_{i+1}\right\|\left\|y_{i}\right\|-\operatorname{ch}\left\|y_{i}\right\|\left\|y_{i+1}\right\|^{2},
$$

or equivalently

$$
\left\|y_{i+1}\right\| \leq \frac{\left\|y_{i}\right\|}{1+c h\left\|y_{i}\right\|}=\frac{1}{\left\|y_{i}\right\|^{-1}+c h}
$$

where $\|y\|=\sqrt{y^{\top} P y}$. The latter inequality immediately implies (see the motivating example in the introduction) that

$$
\left\|y_{i}\right\| \leq \frac{1}{i c h} \quad \text { for } \quad i=1,2, \ldots
$$

independently of the initial state $y_{0}$.

3) Approximation property. In view of Lemma 3.9, the function $\tilde{f}$ is uniformly continuous on $S$.

Let $0<r_{1}<1<r_{2}$ be arbitrary numbers $y_{i}, y_{i+1} \in K\left(r_{1}, r_{2}\right)$, where the set $K\left(r_{1}, r_{2}\right)$ is defined in Proposition 3.7. According to Proposition 3.7 the function is uniformly continuous on $K\left(r_{1}, r_{2}\right)$. Let $\phi\left(\cdot, y_{i}\right)$ be a solution to (5.4) with the initial condition $y(0)=y_{i}$. Then

$$
\begin{gathered}
\left\|\tilde{\phi}\left(h, y_{i}\right)-y_{i+1}\right\|= \\
\left\|\int_{0}^{h}\right\| \tilde{\phi}\left(\tau, y_{i}\right)\left\|^{2} \tilde{f}\left(\tilde{\phi}\left(\tau, y_{i}\right)\right)-\right\| y_{i}\|\| y_{i+1}\left\|\tilde{f}\left(y_{i+1}\right) d \tau\right\| \leq \\
\int_{0}^{h}\left\|\tilde{\phi}\left(\tau, y_{i}\right)\right\|^{2}\left\|\tilde{f}\left(\tilde{\phi}\left(\tau, y_{i}\right)\right)-\tilde{f}\left(y_{i+1}\right)\right\| d \tau \\
+\int_{0}^{h}\left|\left\|\tilde{\phi}\left(\tau, y_{i}\right)\right\|^{2}-\left\|y_{i}\right\|\left\|y_{i+1}\right\|\right|\left\|\tilde{f}\left(y_{i+1}\right)\right\| d \tau .
\end{gathered}
$$

Taking into account that $\left\|\tilde{\phi}\left(\tau, y_{i}\right)\right\|^{2}=V\left(\tilde{\phi}\left(\tau, y_{i}\right)\right)$ and $V$ is a Lyapunov function, we conclude $\left\|\tilde{\phi}\left(\tau, y_{i}\right)\right\|^{2} \leq V\left(y_{i}\right)=\left\|y_{i}\right\|^{2}$ and

$$
\begin{gathered}
\left\|\tilde{\phi}\left(h, y_{i}\right)-y_{i+1}\right\| \leq\left\|y_{i}\right\|^{2} \int_{0}^{h}\left\|\tilde{f}\left(\tilde{\phi}\left(\tau, y_{i}\right)\right)-\tilde{f}\left(y_{i+1}\right)\right\| d \tau \\
\quad+f^{\max }\left\|y_{i}\right\| \int_{0}^{h}\left\|\tilde{\phi}\left(\tau, y_{i}\right)-y_{i}\right\| d \tau \\
+f^{\max }\left\|y_{i}\right\| \int_{0}^{h}\left\|\tilde{\phi}\left(\tau, y_{i}\right)-y_{i+1}\right\| d \tau
\end{gathered}
$$


where $f^{\max }=\sup _{y \in K\left(r_{1}, r_{2}\right)} \tilde{f}(y)$. Repeating the arguments given in the proof of Theorem 4.1, we derive the existence of the desired estimate (2.6).

The conclusions about the existence of a consistent implicit discretization given in Section 4, can be expanded to systems with positive homogeneity degrees, provided that the condition (5.1) holds. This condition is required for the existence of the semi-implicit discretization. In particular it holds if $W_{\alpha}$ is the invertible map on $\mathbb{R}^{n}$.

Remark 5.2. If $n \geq 3, \tilde{f} \in C^{1}\left(\mathbb{R}^{n} \backslash\{\mathbf{0}\}\right)$ and

$$
\operatorname{det}\left(I-\alpha \frac{\partial}{\partial x}(\|x\| \tilde{f}(x))\right) \neq 0, \quad \forall x \in S, \quad \forall \alpha>0,
$$

then the condition (5.1) holds. This immediately follows from [49], Theorem 1.1 and homogeneity of $\|\cdot\| \tilde{f}(\cdot)$.

6. Examples. In this section we consider two examples. The first one is scalar and devoted to consistent discretization of a fixed-time stable sliding mode control algorithm. The control law combines two scalar homogeneous functions $\operatorname{sgn}(x)$ and $|x| x$ to guarantee global fixed-time stabilization of the simplest plant modeled by a single integrator. The second example studies homogeneous single input control system in $\mathbb{R}^{n}$. It demonstrates the possibility of the design of consistent discretizations in more general cases.

6.1. Consistent Discretization of Fixed-Time Control in $\mathbb{R}$. Let us consider the scalar sliding mode control system

$$
\dot{x}=u, \quad x \in \mathbb{R}
$$

with

$$
u=\left\{\begin{array}{ccc}
-|x| x & \text { if } & |x|>1 \\
-\operatorname{sgn}(x) & \text { if } & |x| \leq 1
\end{array}\right.
$$

The control is designed by means of combination of two homogeneous systems with positive and negative degrees. The closed-loop system is globally fixed-time stable [13], i.e., it is finitetime stable with the globally bounded settling time $T\left(x_{0}\right) \leq T^{\max }$. We have $T^{\max }=2$ for the considered scalar control system.

Theorems 5.1 and 4.1 can be directly applied to subsystems

$$
\dot{x}=-x|x|, \quad|x|>1 \quad \text { and } \quad \dot{x}=-\operatorname{sgn}(x), \quad|x| \leq 1,
$$

which are $\mathbf{d}$-homogeneous of degree -1 and 1 , respectively, with $\mathbf{d}(s)=e^{G_{\mathbf{d}} s}, s \in \mathbb{R}$ and $G_{\mathbf{d}}=1$. Applying the consistent discretization schemes to these subsystems we derive

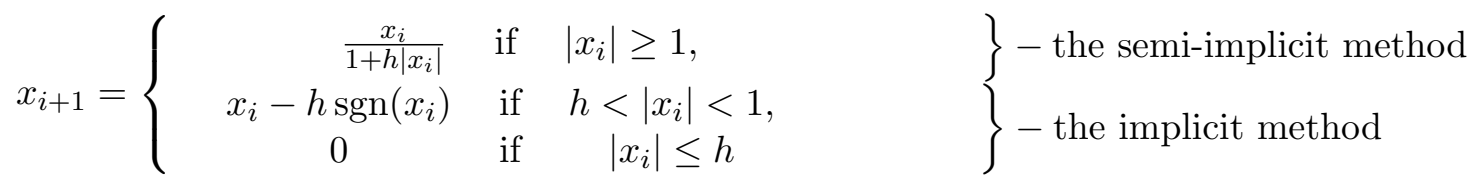

where $h>0$ is the sampling period, $i=0,1, \ldots$. The obtained discretized model is fixed-time stable, i.e., its trajectories converge to zero in $N\left(x_{0}\right)$ samplings steps, and $N\left(x_{0}\right)$ is globally bounded. Since $N_{1}\left(x_{0}\right)$ steps $\left(N_{1} \leq 1 / h\right)$ are needed to guarantee $|x(t h)|=\left|x_{i}\right| \leq 1$ for all 
$i \geq N_{1}$ provided that $\left|x_{0}\right|>1$, and $N_{2}\left(x_{0}\right)$ steps $\left(N_{2} \leq 1 / h\right)$ are needed to reach the origin if $\left|x_{0}\right| \leq 1$, then $N \leq N_{1}+N_{2}$ and

$$
N\left(x_{0}\right) \leq \frac{2}{h}
$$

Notice that if the control law is implemented in a sampled way as

$$
u(t)=u_{i}, \quad t \in[i h,(i+1) h),
$$

then

$$
x(t)=x_{i}+(t-h) u_{i} \quad t \in[i h,(i+1) h) .
$$

In order to guarantee $x((i+1) h)=x_{i+1}$ the control values $u_{i}$ should be selected as follows

$$
u_{i}=\left\{\begin{array}{clc}
-\frac{\left|x_{i}\right| x_{i}}{1+h\left|x_{i}\right|} & \text { if } \quad\left|x_{i}\right| \geq 1, \\
-\operatorname{sgn}\left(x_{i}\right) & \text { if } \quad h<\left|x_{i}\right|<1, \\
-\frac{x_{i}}{h} & \text { if } \quad\left|x_{i}\right| \leq h,
\end{array}\right\} \text { - the semi-implicit method }
$$

which correspond to the semi-implicit $u_{i}=-\left|x_{i}\right| x_{i+1}$ and the implicit $u_{i} \in-\operatorname{sgn}\left(x_{i+1}\right)$ discretizations of the control law, respectively. Therefore, the closed-loop system with the sampled control is also fixed-time stable, i.e., $x(t)=0$ for $t \geq h N\left(x_{0}\right)$. This perfectly corresponds to the continuous-time case. Since $h N\left(x_{0}\right) \rightarrow T\left(x_{0}\right)$ as $h \rightarrow 0^{+}$, we conclude that the same settling time estimate $h N\left(x_{0}\right) \leq T_{\max }=2$ holds even for the system with the sampled control actions.

6.2. Consistent Discrete-time Approximation of a Homogeneous System in $\mathbb{R}^{n}$. Let us consider the homogeneous control system [11], [38]

$$
\dot{x}=f(x):=A x+B u, \quad A=\left(\begin{array}{ccccc}
0 & 1 & 0 & \ldots & 0 \\
0 & 0 & 1 & \ldots & 0 \\
\ldots & \ldots & \ldots & \ldots & \ldots \\
0 & 0 & 0 & \ldots & 1 \\
0 & 0 & \ldots & 0
\end{array}\right), \quad B=\left(\begin{array}{c}
0 \\
0 \\
\ldots \\
0 \\
1
\end{array}\right),
$$

where $x=\left(x_{1}, x_{2}, \ldots, x_{n}\right)^{\top}$,

$$
\begin{aligned}
& u=u_{\nu}(x):=\|x\|_{\mathbf{d}}^{\frac{(n+1)(\nu+1)}{2}} \sum_{j=1}^{n} \frac{k_{j} x_{j}}{\|x\|_{\mathbf{d}}^{\frac{(n+1)(1-\nu)}{2}+j \nu}}, \\
& K=\left(\begin{array}{llll}
k_{1} & k_{2} & \ldots & k_{n}
\end{array}\right) \in \mathbb{R}^{n}, \quad \nu \in\{-1,1\}
\end{aligned}
$$

and $\|\cdot\|_{\mathbf{d}}: \mathbb{R}^{n} \rightarrow \mathbb{R}_{+}$is the canonical d-homogeneous norm for the dilation $\mathbf{d}(s)=e^{s G_{\mathbf{d}}}, s \in \mathbb{R}$ with

$$
G_{\mathbf{d}}=\left(\begin{array}{cccc}
\frac{(n+1)(1-\nu)}{2}+\nu & 0 & \cdots & 0 \\
0 & \frac{(n+1)(1-\nu)}{2}+2 \nu & \cdots & 0 \\
\cdots & 0 & \cdots & \frac{(n+1)(1-\nu)}{2}+n \nu
\end{array}\right)
$$

One has $u_{\nu}(\mathbf{d}(s) x)=e^{\frac{(n+1)(\nu+1)}{2}} u_{\nu}(x)$ and the system is homogeneous of degree $\operatorname{deg}_{\mathbb{F}_{\mathbf{d}}}(f)=\nu$ :

$$
f(\mathbf{d}(s) x)=A \mathbf{d}(s) x+B u_{\nu}(\mathbf{d}(s) x)=e^{\nu s} \mathbf{d}(s)\left(A x+B u_{\nu}(x)\right)=e^{\nu s} \mathbf{d}(s) f(x), \quad x \in \mathbb{R}^{n} \backslash\{\mathbf{0}\} .
$$


The equivalent transformed homogeneous system with $y=\|x\|_{\mathbf{d}} \mathbf{d}\left(-\ln \|x\|_{\mathbf{d}}\right) x$ has the form

$$
\dot{y}=\|y\|^{1+\nu}\left(\frac{\left(I_{n}-G_{\mathbf{d}}\right) y y^{\top} P}{y^{\top} P G_{\mathbf{d}} y}+I_{n}\right) f\left(\frac{y}{\|y\|}\right),
$$

where

$$
f\left(\frac{y}{\|y\|}\right)=\frac{1}{\|y\|}\left(\begin{array}{c}
y_{2} \\
\ldots \\
y_{n-1}^{n-1} \\
\sum_{j=1}^{n} k_{j} y_{j}
\end{array}\right)=(A+B K) \frac{y}{\|y\|},
$$

and $\|y\|=\sqrt{y^{\top} P y}$ with $P$ satisfying (3.2).

Let the gain vector $K$ and a positive definite matrix $P \succ 0$ be selected as follows:

$$
\left(A+B K+G_{\mathbf{d}}\right)^{\top} P+P\left(A+B K+G_{\mathbf{d}}\right)=0, \quad P G_{\mathbf{d}}+G_{\mathbf{d}}^{\top} P \succ 0 .
$$

Such a selection is always possible [50]. In this case, we derive

$$
\begin{gathered}
\tilde{f}(y):=\left(\frac{\left(I_{n}-G_{\mathbf{d}}\right) y y^{\top} P}{y^{\top} P G_{\mathbf{d}} y}+I_{n}\right)(A+B K) \frac{y}{\|y\|}=\frac{1}{\|y\|} \frac{\left(I_{n}-G_{\mathbf{d}}\right) y y^{\top} P(A+B K) y}{y^{\top} P G_{\mathbf{d}} y}+(A+B K) \frac{y}{\|y\|}= \\
\frac{1}{\|y\|} \frac{\left(I_{n}-G_{\mathbf{d}}\right) y\left(-y^{\top} P G_{\mathbf{d}} y\right)}{y^{\top} P G_{\mathbf{d}} y}+(A+B K) \frac{y}{\|y\|}=\left(A+B K+G_{\mathbf{d}}-I_{n}\right) \frac{y}{\|y\|} \cdot
\end{gathered}
$$

Case $\nu=-1$.

In the case of homogeneous system with negative degree we apply Theorem 4.1. The implicit discretization scheme (4.2) has the following representation

$$
y_{i} \in y_{i+1}+h\left(I_{n}-\tilde{A}\right) \tilde{F}\left(y_{i+1}\right), \quad h>0, i=0,1,2, \ldots
$$

where $\tilde{A}=A+B K+G_{\mathbf{d}}$ such that $\tilde{A}^{\top} P+P \tilde{A}=0$ and

$$
\tilde{F}(y)=\left\{\begin{array}{clc}
\left\{\frac{y}{\|y\|}\right\} & \text { if } & y \neq 0 \\
\mathcal{B} & \text { if } & y=0,
\end{array}\right.
$$

where $\mathcal{B}$ is the unit ball in $\mathbb{R}^{n}$ with the norm $\|y\|=\sqrt{y^{\top} P y}$. Notice that the condition (6.3) implies that $I_{n}-\tilde{A}$ is invertible.

Let us denote $q_{i+1}=\left\|y_{i+1}\right\|$ and $z_{i+1}=\frac{y_{i+1}}{\left\|y_{i+1}\right\|}$. Then the inclusion (6.4) has the following solution

- if $y_{i}^{\top}\left(I_{n}-\tilde{A}\right)^{-\top} P\left(I_{n}-\tilde{A}\right)^{-1} y_{i} \leq h^{2}$ then

$$
q_{i+1}=0 \quad \text { and } \quad z_{i+1}=h^{-1}\left(I_{n}-h \tilde{A}\right)^{-1} y_{i}
$$

- otherwise, $q_{i+1}$ and $z_{i+1}$ are derived as the solution to

$$
\left(\left(q_{i+1}+h\right) I_{n}-h \tilde{A}\right) z_{i+1}=y_{i}, \quad z_{i+1}^{\top} P z_{i+1}=1,
$$

where $y_{i}=\left\|x_{i}\right\|_{\mathbf{d}} \mathbf{d}\left(-\ln \left\|x_{i}\right\|_{\mathbf{d}}\right) x_{i}$. Solution to (6.6) always exists due to Theorem 4.1. To find it the equation

$$
y_{i}^{\top}\left(\left(q_{i+1}+h\right) I_{n}-h \tilde{A}\right)^{-\top} P\left(\left(q_{i+1}+h\right) I_{n}-h \tilde{A}\right)^{-1} y_{i}=1,
$$


that is polynomial with respect to $q_{i+1}$, must be initially solved. For $n=2$ the system (6.6) implies a quartic equation with respect to $q_{i+1}$, so it can be solved explicitly using Ferrari formulas. In other cases some proper computational procedure can be utilized. The simulation results for $n=2, \nu=-1, x_{0}=\left(\begin{array}{ll}0.2247 & 0.4494\end{array}\right)^{\top}$ and

$$
P=\left(\begin{array}{ll}
9.1050 & 1.7829 \\
1.7829 & 0.8914
\end{array}\right), K=\left(\begin{array}{ll}
-10.2139 & -3.0000
\end{array}\right)
$$

are given in Fig. 6.1a and Fig. 6.1b, where the developed consistent discretization scheme is compared with the explicit Euler scheme. The simulations confirm finite-time convergence of $\left\{x_{i}\right\}$ to zero in a finite number of steps for the consistent discrete-time model, where

$$
x_{i}=\mathbf{d}\left(\ln \left\|y_{i}\right\|\right) \frac{y_{i}}{\left\|y_{i}\right\|}
$$

and $\left\{y_{i}\right\}$ is the solution to (6.4), while the system obtained using the explicit Euler discretization is not even asymptotically stable (see Fig. 6.1b).

Case $\nu=1$.

In this case we use Theorem 5.1. The semi-implicit discretization (5.3) gives

$$
\left(I_{n}+h\left\|y_{i}\right\|\left(I_{n}-\tilde{A}\right)\right) y_{i+1}=y_{i}, \quad i=0,1,2, \ldots
$$

The matrix $\left(I_{n}+h\left\|y_{i}\right\|\left(I_{n}-\tilde{A}\right)\right)$ has only positive eigenvalues for any $h>0$ and any $\left\|y_{i}\right\|$ (since $\tilde{A}^{\top} P+P \tilde{A}=0$ ), then it is invertible, so

$$
y_{i+1}=\left(I_{n}+h\left\|y_{i}\right\|\left(I_{n}-\tilde{A}\right)\right)^{-1} y_{i}
$$

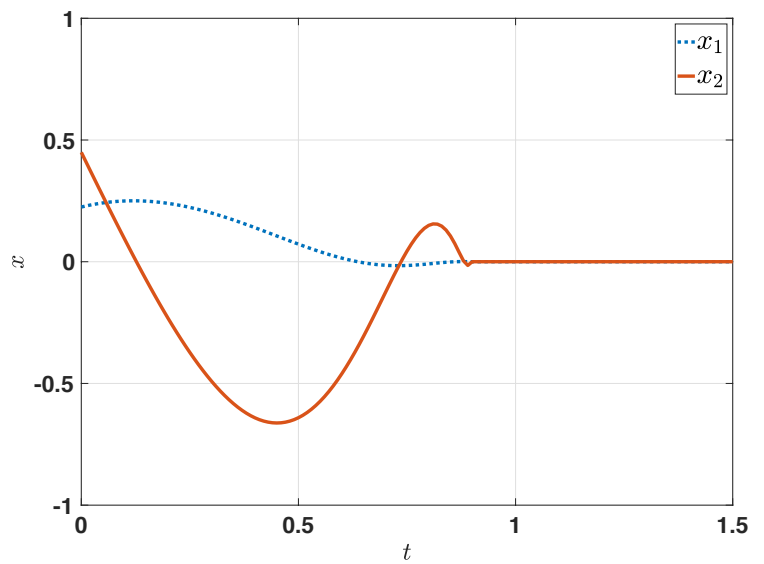

(a) The consistent discrete-time model (6.4)

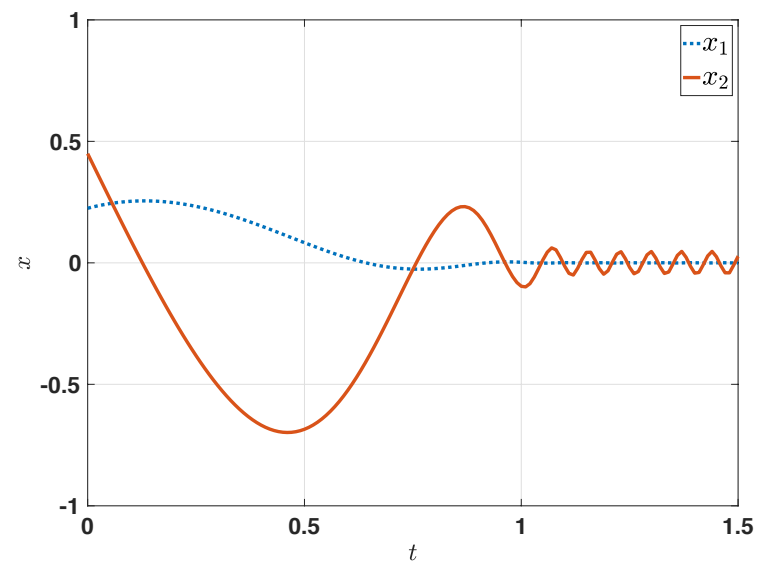

(b) The explicit Euler scheme $x_{i+1}=x_{i}+h f\left(x_{i}\right)$

Figure 6.1: Comparison of the discrete-time models for $h=0.01$ and $\nu=-1$. 
The results of the numerical simulation for $\nu=1, n=2, x_{0}=\left(\begin{array}{ll}10.5607 & 0\end{array}\right)^{\top}$ and

$$
P=\left(\begin{array}{ll}
3.6173 & 2.6173 \\
2.6173 & 2.6173
\end{array}\right), K=\left(\begin{array}{ll}
-1.3821 & -3.0000
\end{array}\right)
$$

are presented in Fig. 6.2a and Fig. 6.2b, where the developed consistent discretization scheme is compared with the explicit Euler scheme.

The simulations show an oscillatory behavior of the discrete-time model obtained using the explicit Euler scheme for $h \in(0.03,0.06)$ (see Fig. 6.2b). For $h>0.06$ the explicit scheme was found to be unstable (solution blows up for the given $x_{0}$ ).

The consistency of the discrete-time model (6.8), (6.7) obtained using the proposed discretization scheme (5.3) is also confirmed by numerical experiments. The practical fixed-time stability is observed in simulations even for large sampling periods (up to $h=1$ ) and large initial conditions (up to $10^{20}$ ).

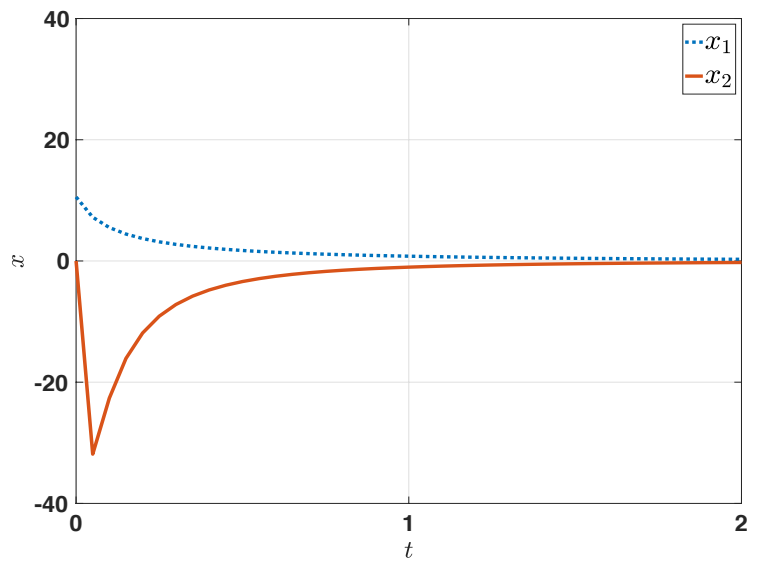

(a) The consistent discrete-time model (6.8)

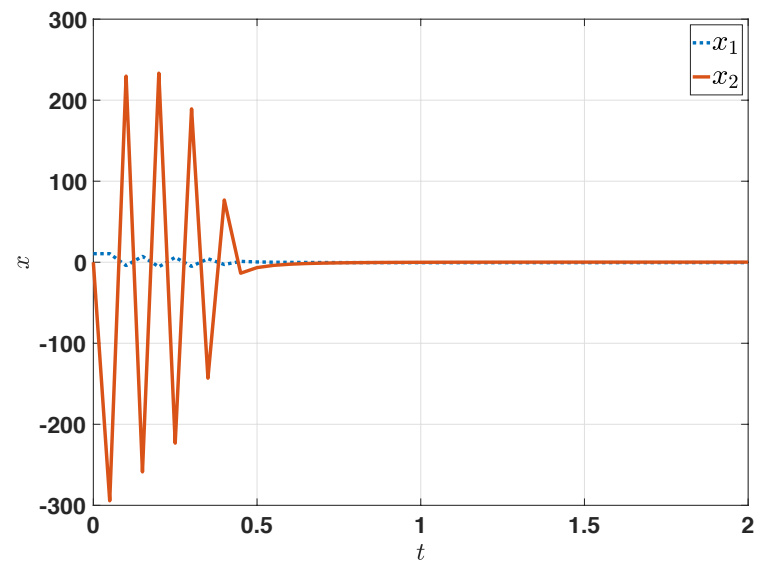

(b) The explicit Euler scheme $x_{i+1}=x_{i}+h f\left(x_{i}\right)$

Figure 6.2: Comparison of the discrete-time models for $h=0.05$ and $\nu=1$.

7. Conclusions and Discussions. The problem of a consistent discrete-time approximation of finite-time and fixed-time stable systems is studied. It is shown that any stable homogeneous system with negative degree admits an implicit discretization preserving finite-time stability property. The topological equivalence of a homogeneous stable system to a quadratically stable one, is utilized for the design of this scheme. Similarly, the consistent semi-implicit discretization is developed for homogeneous systems with positive degree.

The obtained discretization scheme can be utilized for numerical simulations of finitetime and fixed-time stable homogeneous ODEs. It is also useful for digital (sampled-time) implementation of finite-time or fixed-time controllers/observers. Indeed, let us consider again the example given in the previous section (Case $\nu=-1)$. Taking into account $x=\mathbf{d}(\ln \|y\|) \frac{y}{\|y\|}$ and $u_{\nu}(\mathbf{d}(s) x)=u_{\nu}(x)$ for $\nu=-1$, we derive that the implicit discretization of the control 
law (6.2) can be defined as follows

$$
u_{\nu}\left(x_{i+1}\right)=u_{\nu}\left(\frac{y_{i+1}}{\left\|y_{i+1}\right\|}\right)=u_{\nu}\left(z_{i+1}\right)=K z_{i+1},
$$

where $z_{i+1}$ is given by (6.5) or (6.6). According to the conventional implicit discretization technique [1], [4], [17], [18] this value is suggested to be selected for the time interval $\left[t_{i}, t_{i+1}\right)$ during digital implementation of the control law (6.2) in the system (6.1), i.e.,

$$
u(t)=u_{i}:=K z_{i+1}, \quad t \in[i h,(i+1) h) .
$$

The solution to the system (6.1) in this case is given by

$$
x(t)=e^{A t} x_{i}+\int_{0}^{t} e^{A(t-s)} B u_{i} d s, \quad t \in[i h,(i+1) h), \quad x_{i}:=x(i h) .
$$

The implicit sampled control (7.1) completely rejects the numerical chattering (see Fig. 8.1a and 8.1b), which always exists for the explicit implementation of the control law (6.2) (see Fig. 8.2a and 8.2b). In the simplest scalar cases (see Sections 1.1 and 6.1) such an approach allows us to design finite-time and fixed-time stabilizing sampled control algorithms. The development of consistent digital implementations of high-order homogeneous controllers and observers in the general case is an important problem for future research.

The methodology of a consistent discretization could also be expanded to systems with sliding modes, which are discontinuous on manifolds (but not only in the origin as in the present paper), in particular, to homogeneous high order sliding mode systems [51].

8. Acknowledgments. The authors thank the support of the French National Research Agency (ANR), Project ANR-18-CE40-0008 "DIGITSLID". The first and the second authors also acknowledge the support of the Government of Russian Federation (Grant 08-08) and the Ministry of Education and Science of Russian Federation (Project 14.Z50.31.0031).

\section{REFERENCES}

[1] O. Huber, V. Acary, and B. Brogliato, "Lyapunov stability and performance analysis of the implicit discrete sliding mode control." IEEE Transactions on Automatic Control, vol. 61, no. 10, pp. 30163030, 2016.

[2] V. I. Utkin, Sliding Modes in Control Optimization. Berlin: Springer-Verlag, 1992.

[3] A. Levant, "On fixed and finite time stability in sliding mode control," in IEEE Conference on Decision and Control, 2013, pp. 4260-4265.

[4] V. Acary, B. Brogliato, and Y. Orlov, "Chattering-free digital sliding-mode control with state observer and disturbance rejection," IEEE Transactions on Automatic Control, vol. 57, no. 5, pp. 1087-1101, 2012.

[5] R. Kikuuwe, S. Yasukouchi, H. Fujimoto, and M. Yamamoto, "Proxy-based sliding mode control: A safer extension of pid position control," IEEE Transactions on Robotics, vol. 26, no. 4, pp. 670-683, 20110.

[6] O. Huber, B. Brogliato, V. Acary, A. Boubakir, F. Plestan, and B. Wang, Recent Trends in Sliding Mode Control. IET, London, 2016, ch. Experimental results on implicit and explicit time-discretization of equivalent control-based sliding mode control, pp. 207-235.

[7] B. Wang, B. Brogliato, V. Acary, A. Boubakir, and F. Plestan, "Experimental comparisons between implicit and explicit implementations of discrete-time sliding mode controllers: Toward input and output chattering suppression," IEEE Transactions on Control Systems Technology, vol. 23, no. 5, pp. 2071-2075, 2015. 


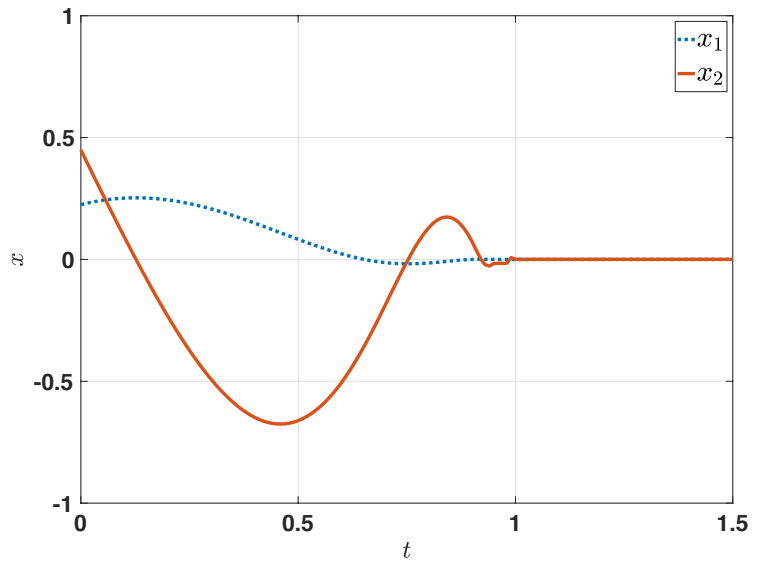

(a) System states

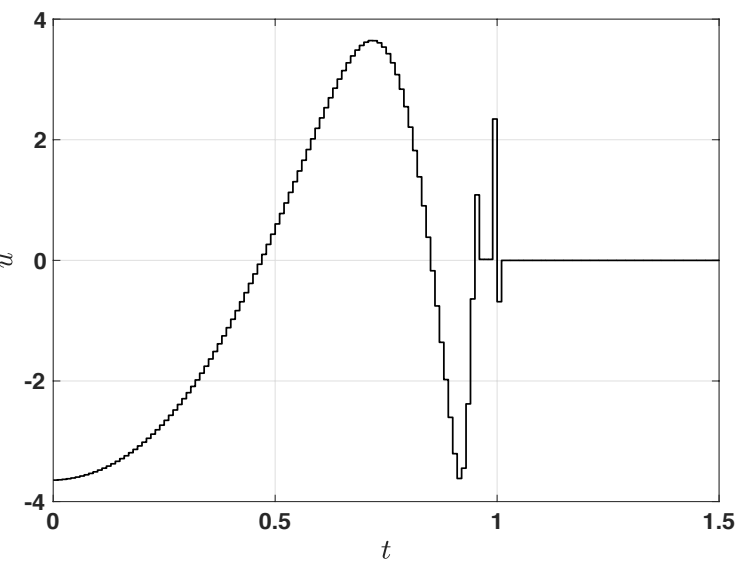

(b) Control signal

Figure 8.1: Evolution of the system (7.2) with $u_{i}=K z_{i+1}, h=0.01$ and $\nu=-1$.

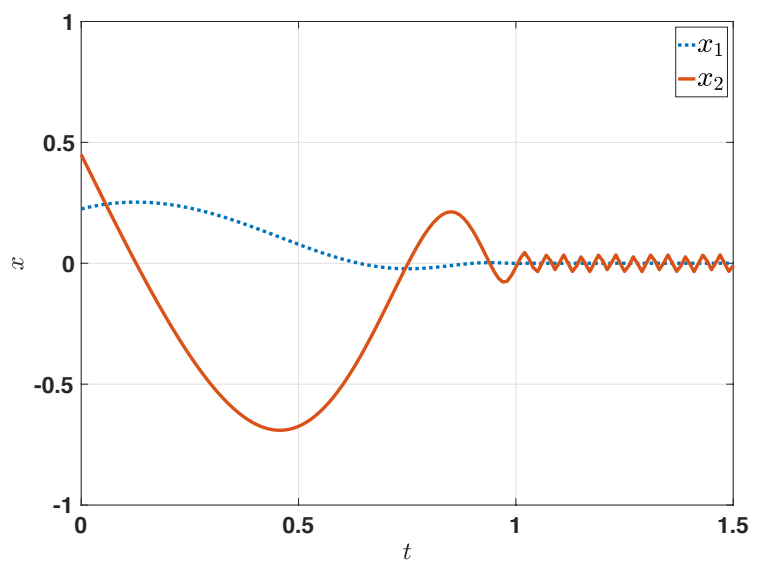

(a) System states

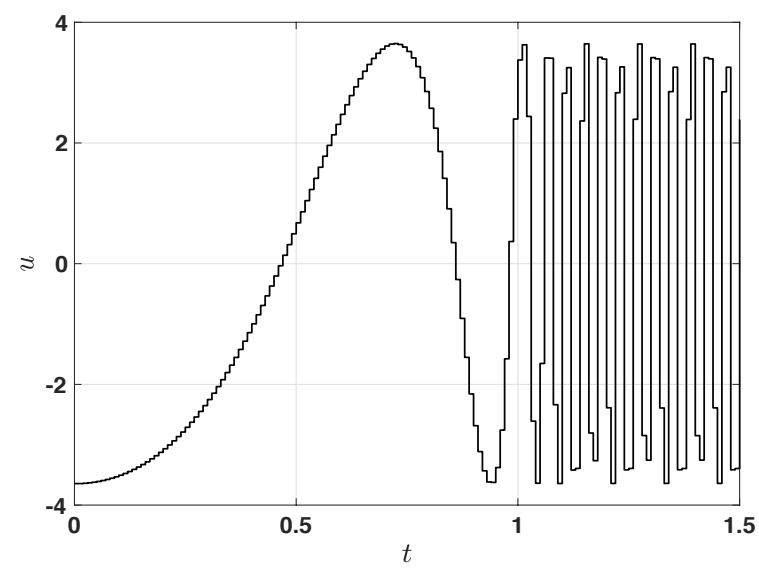

(b) Control signal

Figure 8.2: Evolution of the system (7.2) with $u_{i}=u_{\nu}\left(x_{i}\right), h=0.01$ and $\nu=-1$.

[8] A. Levant, "Sliding order and sliding accuracy in sliding mode control," International Journal of Control, vol. 58, no. 6, pp. 1247-1263, 1993.

[9] S. Bhat and D. Bernstein, "Finite time stability of continuous autonomous systems," SIAM J. Control Optim., vol. 38, no. 3, pp. 751-766, 2000.

[10] V. Andrieu, L. Praly, and A. Astolfi, "Homogeneous Approximation, Recursive Observer Design, and Output Feedback," SIAM Journal of Control and Optimization, vol. 47, no. 4, pp. 1814-1850, 2008.

[11] A. Polyakov, D. Efimov, and W. Perruquetti, "Finite-time and fixed-time stabilization: Implicit Lyapunov function approach," Automatica, vol. 51, no. 1, pp. 332-340, 2015.

[12] F. Lopez-Ramirez, A. Polyakov, D. Efimov, and W. Perruquetti, "Finite-time and fixed-time observer design: Implicit Lyapunov function approach," Automatica, vol. 87, no. 1, pp. 52-60, 2018.

[13] A. Polyakov, "Nonlinear feedback design for fixed-time stabilization of linear control systems," IEEE Transactions on Automatic Control, vol. 57(8), pp. 2106-2110, 2012. 
[14] Z. Galias and X. Yu, "Complex discretization behaviours of a simple sliding-mode control system," IEEE Transactions on Circuits and Systems-II: Express Briefs,, vol. 53, no. 8, pp. 652-656, 2006.

[15] B. Brogliato and A. Polyakov, "Globally stable implicit Euler time-discretization of a nonlinear singleinput sliding-mode control system," in Proc. IEEE Conference on Decision and Control, Osaka, Japan, 2015, pp. 5426-5431.

[16] D. Efimov, A. Polyakov, A. Levant, and W. Perruquetti, "Realization and discretization of asymptotically stable homogeneous systems," IEEE Transactions on Automatic Control, vol. 62, no. 11, pp. 59625969, 2017.

[17] F. Miranda-Villatoro, B. Brogliato, and F. Castanos, "Multivalued robust tracking control of Lagrange systems: Continuous and discrete-time algorithms," IEEE Transactions on Automatic Control, vol. 62 , no. 9, pp. 4436-4450, 2017.

[18] — - "Set-valued sliding-mode control of uncertain linear systems: Continuous and discrete-time analysis," SIAM Journal of Control and Optimization, vol. 56, no. 3, pp. 1756-1793, 2018.

[19] V. Zubov, "On systems of ordinary differential equations with generalized homogenous right-hand sides," Izvestia vuzov. Mathematica., vol. 1, pp. 80-88, 1958 (in Russian).

[20] H. Hermes, "Nilpotent approximations of control systems and distributions," SIAM Journal of Control and Optimization, vol. 24, no. 4, p. 731, 1986.

[21] L. Rosier, "Etude de quelques problèmes de stabilization," PhD Thesis, Ecole Normale Superieure de Cachan (France), 1993.

[22] L. Grune, "Homogeneous state feedback stabilization of homogeneous systems," SIAM Journal of Control and Optimization, vol. 38, no. 4, pp. 1288-1308, 2000.

[23] A. Polyakov, D. Efimov, E. Fridman, and W. Perruquetti, "On homogeneous distributed parameters equations," IEEE Transactions on Automatic Control, vol. 61, no. 11, pp. 3657-3662, 2016.

[24] A. Aleksandrov, A. Platonov, and Y. Chen, "On stability of difference schemes for a class of nonlinear switched systems," Transactions on Computational Sciences XXVII, pp. 53-67, 2016.

[25] V. I. Zubov, Methods of A.M. Lyapunov and Their Applications. Noordhoff, Leiden, 1964 (Translated from Russian: V.I. Zubov, Metody Lyapunova i ih primenenie, Leningrad: LGU, 1957).

[26] W. Hahn, Stability of Motion. New York: Springer-Verlag Berlin Heidelberg, 1967.

[27] A. Andreini, A. Bacciotti, and G. Stefani, "Global stabilizability of homogenenous vector fields of odd degree," Systems \& Control Letters, vol. 10, pp. 251-256, 1988.

[28] Y. Orlov, "Finite time stability and robust control synthesis of uncertain switched systems," SIAM Journal of Control and Optimization, vol. 43, no. 4, pp. 1253-1271, 2005.

[29] A. Levant, "Homogeneity approach to high-order sliding mode design," Automatica, vol. 41, no. 5, pp. 823-830, 2005.

[30] M. Kawski, "Geometric homogeneity and stabilization," in Proc. IFAC Nonlinear Control Symposium, A. Krener and D. Mayne, Eds., Lake Tahoe, CA, 1995, pp. 164-169.

[31] L. Rosier, "Homogeneous Lyapunov function for homogeneous continuous vector field," Systems $E$ Control Letters, vol. 19, pp. 467-473, 1992.

[32] W. Perruquetti, T. Floquet, and E. Moulay, "Finite-time observers: application to secure communication," IEEE Transactions on Automatic Control, vol. 53, no. 1, pp. 356-360, 2008.

[33] K. Zimenko, D. Efimov, A. Polyakov, and W. Perruquetti, "A note on delay robustness for homogeneous systems with negative degree," Automatica, vol. 79, no. 5, pp. 178-184, 2017.

[34] J.-B. Pomet and C. Samson, "Time-varying exponential stabilization of nonholonomic systems in power form," Inria, RR-2126, 1993.

[35] F. Dinuzzo and A. Ferrara, "Higher order sliding mode controllers with optimal reaching," IEEE Transactions on Automatic Control, vol. 54, no. 9, pp. 2126-2136, 2009.

[36] A. Levant, "Quasi-continuous high-order sliding-mode controllers," IEEE Transactions on Automatic Control, vol. 50, no. 11, pp. 1812-1816, 2005.

[37] A. Polyakov, "Quadratic-like stabilizability of homogeneous systems," in Proc. Conference on Decision and Control, Melbourne, Australia, 2017, pp. 577-582.

[38] _ - "Sliding mode control design using canonical homogeneous norm," International Journal of Robust and Nonlinear Control, 2018 (DOI: 10.1002/rnc.4058).

[39] S. P. Bhat and D. S. Bernstein, "Geometric homogeneity with applications to finite-time stability," Mathematics of Control, Signals and Systems, vol. 17, pp. 101-127, 2005. 
[40] E. Cruz-Zavala, J. Moreno, and L. Fridman, "Uniform robust exact differentiator," IEEE Transactions on Automatic Control, vol. 56, no. 11, pp. 2727-2733, 2011.

[41] A. F. Filippov, Differential Equations with Discontinuous Righthand Sides. Kluwer Academic Publishers, 1988.

[42] E. Roxin, "On finite stability in control systems," Rendiconti del Circolo Matematico di Palermo, vol. 15, pp. 273-283, 1966.

[43] A. Polyakov and L. Fridman, "Stability notions and Lyapunov functions for sliding mode control systems," Journal of The Franklin Institute, vol. 351, no. 4, pp. 1831-1865, 2014.

[44] A. Polyakov, J.-M. Coron, and L. Rosier, "On homogeneous finite-time control for linear evolution equation in hilbert space," IEEE Transactions on Automatic Control, vol. 63, no. 9, pp. 3143-3150, 2018.

[45] A. Pazy, Semigroups of Linear Operators and Applications to Partial Differential Equations. Springer, 1983.

[46] A. Polyakov, J.-M. Coron, and L. Rosier, "On finite-time stabilization of evolution equations: A homogeneous approach," in Conference on Decision and Control, 2016, pp. 3143-3148.

[47] L. Praly, "Generalized weighted homogeneity and state dependent time scale for linear controllable systems," in Proc. IEEE Conference on Decision and Control, San Diego, USA, 1997, pp. 4342-4347.

[48] S. Kakutani, "A generalization of Brouwer's fixed point theorem," Duke Mathematical Journal., vol. 8, no. 3, pp. 457-459, 1941.

[49] M. Ruzhansky and M. Sugimoto, "On global inversion of homogeneous maps," Bull. Math. Sci., vol. 5, pp. 13-18, 2015.

[50] A. Polyakov, D. Efimov, and W. Perruquetti, "Robust stabilization of MIMO systems in finite/fixed time," International Journal of Robust and Nonlinear Control, vol. 26, no. 1, pp. 69-90, 2016.

[51] A. Levant, "Higher-order sliding modes, differentiation and output-feedback control," International Journal of Control, vol. 76, no. 9-10, pp. 924-941, 2003. 